\title{
Water Memory Due to Chains of Nano-Pearls
}

\author{
Auguste Meessen \\ Université Catholique de Louvain (UCL), Louvain-la-Neuve, Belgium \\ Email: auguste@meessen.net
}

How to cite this paper: Meessen, A. (2018) Water Memory Due to Chains of Nano-Pearls. Journal of Modern Physics, 9, 2657-2724.

https://doi.org/10.4236/jmp.2018.914165

Received: November 20, 2018

Accepted: December 26, 2018

Published: December 29, 2018

Copyright $\odot 2018$ by author and Scientific Research Publishing Inc. This work is licensed under the Creative Commons Attribution International License (CC BY 4.0).

http://creativecommons.org/licenses/by/4.0/

\begin{abstract}
Biologically active molecules create substitutes in liquid water by forming single-domain ferroelectric crystallites. These nanoparticles are spherical and constitute growing chains. The dipoles are aligned, but can be set in oscillation at the frequency of vibration of the charged part of active molecules. They are then automatically trimmed and become information carriers. Moreover, they produce an oscillating electric field, causing autocatalytic multiplication of identical chains in the course of successive dilutions. Active molecules are thus only required to initiate this process. Normally, they excite their specific receptors by resonance, but trimmed chains have the same effect. This theory is confirmed by many measurements.
\end{abstract}

\section{Keywords}

Water Memory, Extra-High Dilutions, Ferroelectric Particles, Chains of Nanoparticles, Water Bridges, Molecular Interactions

\section{Introduction}

The concept of water memory is based on experimental results of measurements, published 30 years ago in the prestigious scientific journal Nature [1]. This astonishing phenomenon had been discovered by Jacques Benveniste and his collaborators, but one month later, the same journal declared that it was a delusion [2]. Even the publication of the discovery was already accompanied by an astounding editorial reservation [3]. The editor in chief, John Maddox, declared indeed that "there is no physical basis for such an activity" and announced even that independent investigators would "observe repetitions of the experiments".

When they arrived at Benveniste's laboratory, it turned out that John Maddox was accompanied by the professional magician James Randi and the debunker of scientific fraud Walter Steward. The objective was thus to detect errors or fraud. During their stay in Paris, the first experiments confirmed the published result, 
but not later ones. The inquisitors did immediately publish a devastating conclusion: the reported results are not reproducible and merely due to imagination [2]. Randi stated even that they should be compared to the sensational claim of having seen a unicorn, where there was merely a goat.

The load of the accusation fell on Jacques Benveniste (1935-2004). He was a medical doctor, who had practiced several years before opting for research. In California, he discovered the platelet-activating factor and determined its role in immunology [4] [5]. He returned to France in 1973 and joined INSERM (National Institute of Health and Medical Research). Since 1980, he directed Unit 200, devoted to research in immunology, allergy and inflammation. He discovered there a very sensitive method to detect allergens. It required careful counting of discolored cells, but Elisabeth Davanas succeeded very well [1]. The young medical doctor Bernard Poitevain, who joined the team to prepare a thesis, asked somewhat later if he could test the efficiency of homeopathic dilutions by means of this method. Benveniste answered: "try if you want, but there will be no effect; high dilutions are merely water" ([6], p. 45). Nevertheless, the results were positive and confirmed by other members of the group.

This phenomenon was totally unexpected, since successive dilutions of biologically active molecules in pure water do necessarily lead to their complete elimination. Was it really possible to create substitutes that are only constituted of water molecules? Further experimentation did prove that these structures should even be able to mimic active molecules of different types. No one knew how this might be achieved. Benveniste verified therefore if these hypothetical structures could be destroyed. It appeared that after heating extra-high dilutions (EHDs) of histamine during one hour at $70^{\circ} \mathrm{C}$, they had completely lost their biological efficiency. Exposition to ultrasound had the same effect ([6], pp. 53-54). The puzzling phenomenon of "water memory" had even a characteristic property: the biological efficiency of EHDs decreased at first, but increased again and reached a high value after about the $9^{\text {th }}$ decimal dilution. Then it dropped and continued to vary in a quasi-periodic way during successive dilutions.

Since the reality of this phenomenon was tested many times for different substances, Benveniste thought that these facts had to be published, although they were unexplained. He insisted on the observed quasi-periodic variations, by providing two figures [1]. However, Maddox was convinced that the reported results cannot be real. He required independent confirmations. They were provided by other laboratories in Italy, Canada and Israel. Eventually, after two-year long discussions ([6], p. 51), Maddox accepted to publish the article if Benveniste did agree that a team of experts could come to "verify the quality of the experimentation". This was more than bizarre, but Benveniste had nothing to hide. The outcome was the terrible accusation that we mentioned [2]. Benveniste was allowed to add a reply, but could only describe how the investigators had proceeded. They created a climate of "intense and constant suspicion". Actually, Benveniste and his collaborators were treated like "criminals". 
In their report, the group of inquisitors insisted on the variability of the peaks of activity and claimed that measurements had been treated in "disregard of statistical principles". They declared even that "the laboratory has fostered and then cherished a delusion about the interpretation of this data". Melinda Baldwin, lecturer on History of Science at Harvard University, identified the actual cause of this grave incident. Maddox considered that scientific journals are shaping science by controlling its quality [7]. This implies enormous power, but also the danger to defend orthodoxy by condemning any deviation. Baldwin mentioned the quasi-periodic variations of the reported biological efficiency. They were strange, but could have an intrinsic cause, deserving further research.

Benveniste considered that his duty was only to establish the reality of these "unbelievable and fear-provoking" facts, since their meaning and the underlying mechanism could be studied later on ([6], p. 61). This is not at all unusual in science. After the events of 1988, the experiments were repeated many times with the statistician Spira, but the previous results were validated. In the course of further tests, Benveniste made a second discovery. He realized in 1993 that two EHDs were able to perturb one another and thought that this was due to electromagnetic fields. He suspected that these "signals" could play an essential role in the constitution of water memory and did prove that they can be detected, amplified, recorded and transmitted. They seemed to be noise in the frequency domain of audible sound waves (lower that $20 \mathrm{kHz}$ ). However, when pure water was exposed to them, it acquired the same properties as EHDs of the initially dissolved active molecules. This information transfer confirmed that water memory is real, but INSERM did not renew the contract for Unit 200 in 1995. Benveniste pursued his research in a room on the parking of his former institute, with a caravan for storing materials. He focused now his efforts on developing "digital biology".

Alain Kaufmann presented in 1994 basic facts and an analysis of the sociological context [8]. Michel Schiff published the same year a more detailed description of the experiments and denounced the dangers of censorship [9]. Benveniste, who had reluctantly accepted to write the preface of this book, attributed there the radical rejection of available evidence to the following reasons: "It cannot be, since if it were true it would have been found two hundred years ago" and "there is no theory behind it." We can add that the discovery of an anomaly may lead at first to incredulity, but well-documented facts should induce a search of their cause. It might be necessary, indeed, to correct some previous assumptions. Peer evaluation is necessary and useful, but not infallible. Schiff insisted that even scientists can "suppress unwanted knowledge" when it would "shatter their current beliefs". Actually, "the long history of scientific dogmatism shows that today's heresy could well become tomorrow's scientific truth".

Francis Beauvais, a former collaborator of Benveniste, provided in 2007 much more details on events at that time [10]. Yolène Thomas [11] continued research on water memory, but it had to be camouflaged as concerning properties of EHDs. Even the French virologist Luc Montagnier, who received in 1988 the 
Nobel Prize for his contribution to the discovery of the HIV virus, was violently attacked when he resumed the experimental study of water memory. He was motivated by scientific curiosity and the constant need of improved medical treatments, while his opponents negated a priori that EHDs in pure liquid water could modify this solvent. Montagnier presented his experimental method in a documentary, realized in 2014 by French TV [12]. It is also available in English [13] and does clearly demonstrate that water memory involves detectable signals at frequencies like those of audible sound waves $(20-20,000 \mathrm{~Hz})$. Montagnier published in 2009 and 2010 important results concerning water memory [14] [15]. He discovered even that viral DNA sequences can be reproduced by means of transmitted signals when the building blocks are available in pure water. These signals had thus to provide the required master plan and this fact might account for strange resurgences of some sicknesses. The conference of Montagnier at UNESCO in 2014 stressed this fact and other medical applications [16].

Visceral opposition to the concept of water memory was often motivated by fear that it could justify homeopathy. The aim of the present study is merely to find out if water memory is real or not. This has to be viewed as a basic problem for condensed matter physics, since bonds between water molecules are constantly broken by thermal agitation in liquid water at the time scale of picoseconds. Martin Chaplin, specialist of properties of water molecules, proposed therefore in 2007 that water memory could result from creating statistically stable clusters of water molecules [17]. Individual molecules would there be replaced by other ones without affecting the global structure. This hypothesis was the most plausible one, but Martin Chaplin added that "much research work remains to be undertaken if these real and observable facts are to be completely understood".

The structure of this article results from the itinerary that we followed. In Section 2, we examine the internal structure of water molecules and their possible interactions. This leads to the concept of "water pearls". In Section 3, we explain why biologically active molecules can create chains of these nano-pearls and why they account for water memory. Section 4 presents more observational evidence concerning these chains. It is diverse, detailed and very remarkable, but the concept of water pearls accounts for known facts, while the alternative concept of Coherent Domains does not. In Section 5, we insist on the most important consequence of water memory: molecular interactions are not only possible by means of the "key and slot" model of chemical reactions. Intermolecular communications can also result from oscillating electric fields and resonance effects. It will appear once more that "Nature is written in Lingua Mathematica", as Galilei stated already, but we endeavor to be understandable by non-specialists.

\section{Interactions of Water Molecules}

\subsection{Their Structure and the Dipole Approximation}

Martin Chaplin provides detailed information about the internal structure of 
water molecules [18] and various models that have been proposed [19]. The usual "stick and ball model" insists on the chemical composition $\left(\mathrm{H}_{2} \mathrm{O}\right)$, but the protons of both light atoms are deeply embedded in the common electron cloud of the oxygen and hydrogen atoms. Water molecules are thus practically spherical, but at close range, the protons are surrounded by a spherically symmetric excess density of electrons. They are thus equivalent to point-like charges $q \approx$ $e / 3$. The core of the oxygen atom and the remaining part of the electron cloud are equivalent to a central point-like charge $-2 q$.

The kinetic diameter (for collisions) of water molecules in the terrestrial atmosphere is $0.265 \mathrm{~nm} . \mathrm{H}_{2} \mathrm{O}$ molecules are thus smaller than $\mathrm{O}_{2}, \mathrm{~N}_{2}, \mathrm{CO}_{2}$ and $\mathrm{H}_{2}$ [20]. Their size is slightly greater in the liquid state, because of interactions with surrounding water molecules. The average separation of two oxygen atoms is there measurable by means of $\mathrm{x}$-ray diffraction. The resulting diameter is $d=$ $0.275 \mathrm{~nm}$. In the gaseous state, the angle ${ }^{\mathrm{H}} \mathrm{O}^{\mathrm{H}}$ is $104.5^{\circ}$, but it is close to $106^{\circ}$ in the liquid state. Vibrational and rotational spectra of water molecules disclosed that the length of $\mathrm{OH}$ bonds is $\delta \approx 0.095 \mathrm{~nm}$. Thus, $\delta / \mathrm{d} \approx 1 / 3$. Since water molecules behave in the liquid state like hard spheres that can easily roll on one another, we adopt the model of Figure 1. To simplify later calculations, we chose natural units, where $\delta=1$ and $q=1$. The diameter of a water molecule in the liquid state is then $d \approx 2.90$. Since the angle $\varphi=53^{\circ}$, the distance $a=\cos \varphi \approx 0.60$ and $b=\sin \varphi \approx 0.80$. It follows that $\mathrm{a} / \mathrm{d}=1 / 5$.

Because of their internal point-like charges, water molecules are tripoles, but it is customary to replace them by dipoles. They are constituted by the central charge $-2 q$ and a single charge $+2 q$, situated in the middle between the charges $+q$. This dipole is represented in Figure 2 by a red arrow. By definition, the dipole moment is then $p=2 q$ a. The limited validity of this approximation appears when we calculate the electrostatic potential $V(r, \theta)$ at large distances $r$ from the center of the effective dipole. The angle $\theta$ specifies the chosen direction with respect to the axis of the dipole.

The test charge +1 does then "see" the charges along parallel lines, but their distances are slightly different. They are indicated by thin red lines. Adopting also natural units $\left(4 \pi e^{2} / \delta \varepsilon_{o}=1\right)$ for electrostatic potentials, their sum is



Figure 1. Model of water molecules. 


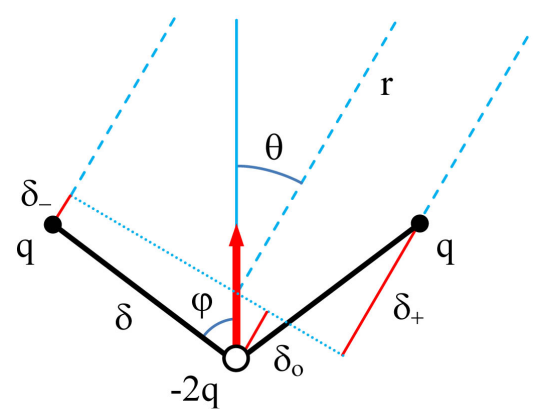

Figure 2. The dipole approximation.

$$
\begin{aligned}
& V(r, \theta)=\frac{-2 q}{r+\delta_{o}}+\frac{q}{r-\delta_{+}}+\frac{q}{r+\delta_{-}} \\
& =\frac{q}{r^{2}}\left(2 \delta_{o}+\delta_{+}-\delta_{-}\right)+\frac{q}{r^{3}}\left(-2 \delta_{o}^{2}+\delta_{+}^{2}+\delta_{-}^{2}\right)+\cdots
\end{aligned}
$$

The first and second order approximations result from $(1+x)^{-1}=1-x+x^{2}$ when $x \ll 1$. Since $\delta_{o}+\delta_{+}=\delta \cos (\varphi-\theta)$ and $\delta_{o}-\delta_{-}=\delta \cos (\varphi+\theta)$, the value of $2 \delta_{o}+\delta_{-}-\delta_{+}=2 \delta \cos \varphi \cos \theta$, where $\delta \cos \varphi=a$. Thus,

$$
V(r, \theta) \approx \frac{p}{r^{2}} \cos \theta, E_{r} \approx \frac{2 p}{r^{3}} \cos \theta, E_{\theta} \approx \frac{-p}{r^{3}} \sin \theta
$$

The radial and angular components of the electric field $E$ at the observation point result from partial derivatives of $V(r, \theta)$. At closer range, there are inevitable corrections. In liquid water, it is also necessary to account for intermediate water molecules, since they are easily reoriented by an applied electric field. The potential $V(r, \theta)$ is then reduced by the factor $1 / \varepsilon_{\mathrm{r}}$, where the relative dielectric constant $\varepsilon_{\mathrm{r}} \approx 80$. When neighboring water molecules are subjected to an electric field, their effective dipoles will be aligned. These molecular chains are broken by thermal agitation when the electric field is extinguished, but they could also be stabilized by association, like sticks in a bundle.

This possibility merits attention, since it is known that electric field lines can be visualized by means of neutral particles, like grains of semolina or short plastic filaments dispersed on oil. The applied electric field does merely polarize these particles, but the induced dipoles tend then to align one another. Could similar chains be formed by means of water molecules? To answer this question, we have to examine all possible types of interactions between water molecules.

\subsection{The Origin of Van der Waals Forces}

Even electrically neutral molecules attract one another in gases, because of Coulomb forces and quantum-mechanical effects. Indeed, if such a particle were subjected to an electric field, it would displace all weakly bound electrons inside this particle. This produces surface charges that create a secondary electric field inside the particle. It opposes displacements of the electrons and would restore neutrality when the applied field is switched off. For oscillating electric fields, this force leads to a resonance for the ensemble of oscillating electrons. That ex- 
plains the appearance of colors and peculiar optical properties of thin granular metal films. They were said to be "anomalous" until they could be explained in terms of collective oscillations of nearly free electrons [21]. Since oscillations of electrons inside neutral particles do also create an oscillating electric field outside these particles, two neutral ones can interact with one another.

Nearly free electrons will be set in coupled oscillations inside neighboring particles. Their resonance frequency is then reduced, but in quantum mechanics $(\mathrm{QM})$, the lowest possible energy of an oscillator is proportional to its resonance frequency. The (zero-point) energy of two neutral particles is thus reduced when they come close enough to one another. This effect can be interpreted as resulting from an attractive force. The existence of this short-range force was discovered by Van der Waals in 1873, since a dense gas does not behave like an ideal one. It corresponds to a model, where velocities are only randomized by collisions of point-like particles, but neutral particles attract already one another at some small distance. The physical origin of this force could only be understood after the development of QM.

Since Van der Waals forces are proportional to the volume of the polarizable particles, they are negligible with respect to other forces for water molecules in the liquid state. However, small metal particles that are suspended in liquid water contain nearly free electrons. They are very polarizable and big enough to attract one another by Van der Waals forces. In liquid water, small metal particles attract thus one another and constitute chains. These "necklaces of pearls" are observable by optical microscopy [22] and attract now much attention, because of expected applications. Similar chains might be relevant for water memory.

\subsection{Hydrogen Bonds and Exchange Effects}

The concept of so-called "hydrogen bonds" was already introduced in 1920, since some quantum effects could be treated in a semi-classical way [23], but simplified models can lead to confusions. The Lewis model, proposed in 1916, was merely based on the fact that many atoms are more stable when their shells contain 2 or 8 electrons. Because of Bohr's semi-classical model of atomic structures and Pauli's exclusion principle, these values correspond to closed shells. Hydrogen atoms contain only 1 electron, while $\mathrm{C}, \mathrm{N}, \mathrm{O}$, and $\mathrm{F}$ atoms do respectively have 4, 5, 6 and 7 electrons in their external shells instead of 8. Molecules like $\mathrm{CH}_{4}, \mathrm{NH}_{3}, \mathrm{OH}_{2}$ and $\mathrm{FH}$ would thus result from the "tendency to complete... the octet of electrons". This lowers the total energy, but $\mathrm{H}_{2} \mathrm{O}$ molecules are special. The left part of Figure 3 represents closed shells of the oxygen atom and the hydrogen atoms by blue rings. The shared electrons are represented by dots, but the oxygen atom is then surrounded by 4 pairs of electrons. There are 2 bound pairs and 2 free pairs.

It was therefore proposed that the negative charge density of the free pairs "might be able to exert sufficient force" on two neighboring oxygen atoms. This would account for mutual attraction of water molecules that allows for structuring of liquid and frozen water. The right part of Figure 3 represents these 



Figure 3. Semi-classical concepts of hydrogen bonds.

"hydrogen bonds" by means of red lines, when neighboring water molecules are assumed to be situated in the same plane. QM revealed that electrons behave according to laws that apply to waves. Every oxygen atom contains two strongly bound electrons and four external electrons in $\left(2 s^{1} 2 p^{3}\right)$ states. Superposition of these wave functions leads to interference effects and the charge distribution of the 4 external electrons acquires then tetragonal symmetry. We might thus think that hydrogen bonds are merely due to stronger electrostatic attraction, but modern biochemistry states that "in a hydrogen bond, a hydrogen atom is shared by two other atoms" [24]. The right part of Figure 3 can then be interpreted in terms of two donor sites of protons and two acceptor sites. This description implies that the intermediate proton might change its position. Hydrogen bonds would then be due to exchange effects, which are also known for nuclear forces.

Figure 4(a) represents the effects of hydrogen bonds between water molecules in $3 \mathrm{D}$ space. The red dots define average positions of the cores of neighboring water molecules in liquid water, while the red lines correspond in a schematic way to electron pairs, but also to possible exchanges of protons between pairs of oxygen atoms. This configuration requires a modification of the internal structure of water molecules, since the normal angular separation of two protons was there $2 \varphi=106^{\circ}$ (Figure 1 ). Here we get 4 equal angles $2 \mu \approx 110^{\circ}$. They are defined by joining the center of the cubic cell to four equally separated vertices. The value of $\cos \mu=h / c=1 / \sqrt{3}$, since $(2 c)^{2}=3(2 h)^{2}$. It follows that $\mu=54.74^{\circ}$.

Figure 4(a) accounts only for one possible lattice structure of ice, since imposed temperatures and pressures can yield different phases for frozen water [25]. In liquid water, adjacent molecules are moving around, since all bonds are constantly broken and reconstituted at an extremely rapid pace [26]. Nevertheless, small-scale order is statistically preserved, while large-scale order is lost. At an intermediate scale, we get the extended lattice-structure of Figure 4(b). Body-centered and empty cubes are alternating. This yields many voids, which will often be filled at higher temperatures. This fact explains the existence of low-density and high density liquid water, as well as analogous amorphous states for frozen water.

It is important to be aware of the quantum-mechanical nature of exchange effects. They are also possible between two $\mathrm{X}$ and $\mathrm{Y}$ atoms, when an intermediate proton could belong to $\mathrm{X}$ or $\mathrm{Y}$. Both possibilities are expressed by the notation XH... Y or X... HY. QM accounts indeed for limited knowledge. The probability distribution for possible positions of electrons is defined by means of their wave functions. Exchange effects are then due to "tunneling" through an intermediate 


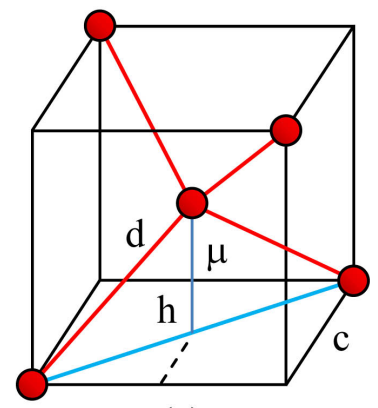

(a)

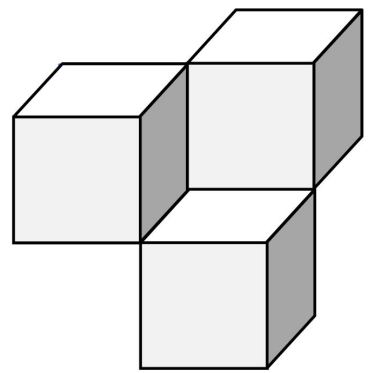

(b)

Figure 4. (a) Idealized model for the relative positions of oxygen atoms in neighboring molecules for ice and liquid water; (b) Extended lattice structure of this type.

potential barrier. This is relevant for bonds between atoms inside molecules and in particular for $\mathrm{H}_{2}^{+}$, where one electron allows for $\mathrm{H}-\mathrm{H}^{+}$or $\mathrm{H}^{+}-\mathrm{H}$. Although protons have a greater mass than electrons, they are also subjected to quantum-mechanical laws. The probability distribution for being at different places in space is then not smeared out, but reduced to needle-like (delta) functions. When a proton has two possible positions, it can be said to be delocalized, but possible exchanges are then not due to tunneling. They result from the fact that the energy of any physical system cannot be precisely determined during short time intervals $\Delta t$. There is always an irreducible uncertainty $\Delta E \approx h / \Delta t$. In semi-classical terms, the proton is able to "jump" over the intermediate potential barrier, when this happens rapidly enough.

\subsection{Coulomb Forces and Exchange Effects for Water Dimers}

To analyze possible effects of protons for interacting water molecules, we begin with the simplest case. Figure 5 represents the cores of two oxygen atoms by open dots, separated by the distance $\mathrm{d}$. The left part of this figure corresponds to purely classical concepts. The intermediate proton has a well-defined position, indicated by a black dot. Since the measured length of $\mathrm{OH}$ bonds is $\delta$, we can calculate the total potential energy $U_{1}$ that results from electrostatic interactions between two point-like particles of charge -2 and one charge +1 . However, the concept of hydrogen bonds means that the intermediate proton has two possible positions. They are represented by gray dots in the upper right part of Figure 5. When they are occupied with equal probabilities, we have to attribute an average charge $+1 / 2$ to these positions. However, even when a particle is delocalized, it cannot exert forces on itself. We will calculate the resulting electrostatic potential energy $U_{2}$. The third configuration would be obtained if it were possible to account for hydrogen bonds in a semi-classical way, by assuming that the proton has only one well-defined position, situated in the middle. This position is represented by a black dot and the potential energy would then be $U_{3}$.

Using natural units for charges, distances and energies, we get

$$
U_{1}=\frac{4}{d}-\frac{2}{\delta}-\frac{2}{d-\delta}=-1.67=U_{2} \text { and } U_{3}=\frac{4}{d}-\frac{2}{d}=-0.69
$$



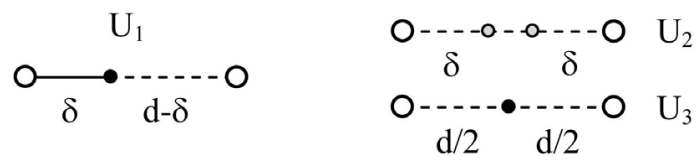

Figure 5. Three conceivable models for O-H-O bonds.

Since $U_{2}=U_{1}$, a strictly classical description is sufficient, although the proton is delocalized. This would even be true (in the present case) if the uncertainty did allow for any partition $q^{\prime}$ and $(1-q)$ of the charge +1 . The third configuration is very unstable and does not account for quantum-mechanical exchange effects. We are now ready to calculate the total potential energies $V_{1}$ to $V_{4}$ for different configurations of dimers, represented in Figure 6. They result from the fact that two water molecules can easily be rotated with respect to one another, but this modifies the potential energy of the interacting tripoles. The usually assumed configuration of $\left(\mathrm{H}_{2} \mathrm{O}\right)_{2}$ is close to the upper left one of Figure 6. The tripole of one molecule is situated in the plane of the drawing and one of its two protons is precisely oriented towards the core of the neighboring molecule. The other tripole is perpendicular to this plane and seen in profile. We will calculate the potential energy $V_{1}$ for the indicated configuration.

$V_{2}$ corresponds to aligned effective dipoles. This configuration would be preferred if water molecules did only contain dipoles, but could even be privileged for effective dipoles, when the dimer is subjected to an electric field. We want thus to see if $V_{2}$ is already close to $V_{1}$ in the absence of an applied electric field. The tripoles should then be orthogonal to one another to minimize repulsion between protons in neighboring molecules. $V_{3}$ is the potential energy for any pair of molecules in a nearly linear chain, where all intermediate protons are ideally situated between two oxygen atoms. The resulting zigzagging configuration is planar and in conformity with a classical representation.

An applied electric field could even allow for a perfectly linear chain, because of intramolecular exchange effects, although this was unknown. For clarity, we consider here two coplanar tripoles. One proton is always situated as close as possible to the core of the neighboring oxygen atom. The other proton has two equally probable positions, above and below the symmetry axis. This would yield the energy $V_{4}$ for any pair of water molecules. Alternatively orthogonal tripoles would reduce repulsion between the delocalized protons. The resulting potential energy is then $V_{5}<V_{4}$.

To facilitate this type of calculations, we note that the total potential energies result always from adding the Coulomb potentials $\left(V=q q^{\prime} / \Delta\right)$ for pairs of point-like charges $q$ and $q$, separated by a distance $\Delta$. We define thus a function $S(x, y, z)$, where $x, y$ and $z$ are differences of Cartesian coordinates, respectively measured towards the right, rear and top. Thus,

$$
V=q q^{\prime} S(x, y, z) \text { where } S(x, y, z)=\left(x^{2}+y^{2}+z^{2}\right)^{-1 / 2}
$$

The values of $V_{1}$ depends on the angle $\varphi=106^{\circ}$ and the complementary angle $\phi=74^{\circ}$. Since $\delta=1$ in natural units, the distance $a_{1}=\cos \phi=0.28$ and $b_{1}=\sin \phi$ 

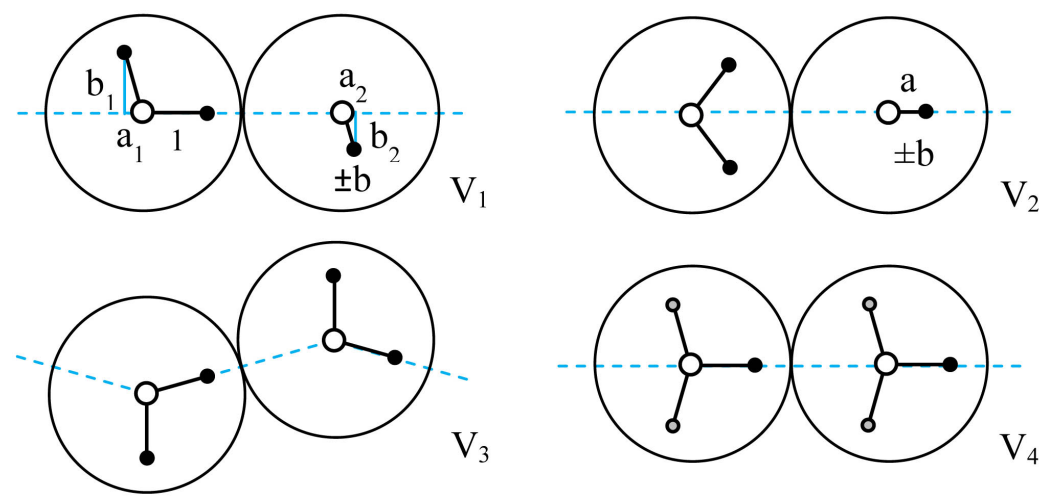

Figure 6. Conceivable structures of water dimers.

$=0.96$. However, $a_{2}=a a_{1}$ and $b_{2}=a b_{1}$, where $a=\cos \varphi=0.60$ and $b=\sin \varphi=$ 0.80 . Thus,

$$
\begin{aligned}
V_{1}= & 4 S(d, 0,0)+2 S\left(d-1+a_{2}, b, b_{2}\right)+2 S\left(d+a_{1}+a_{2}, b, b_{1}+b_{2}\right) \\
& -2 S(d-1,0,0)-4 S\left(d+a_{2}, 0, b_{1}\right)-2 S\left(d+a_{1}, 0, b_{1}\right) \\
= & -0.116 \\
V_{2}= & 4 S(d, 0,0)+4 S(d, b, b)-4 D(d+a, b, 0)-4 D(d-a, 0, b)=-0.093
\end{aligned}
$$

$V_{1}$ can be slightly lowered when the repulsion between the nearest protons is reduced by a small rotation of the left molecule around its center. An angle of $1.4^{\circ}$ is sufficient to reach the minimal potential energy [27]. Since $V_{1}<V_{2}$, it has been assumed that more than 2 water molecules should always be assembled according to the same rule as for the most strongly bound dimer. Water molecules could then only constitute rings or clusters of limited size, but linear polymerization is not excluded for water molecules. It would even be preferred for $n_{2}$ water molecules, compared to clusters of $n_{1}$ molecules, when $\left(n_{2}-1\right) V_{2}<\left(n_{1}-\right.$ 1) $V_{1}$. It is thus sufficient that $n_{2}>1.3 n_{1}$. Of course, long chains of water molecules would be too fragile to resist thermal agitation in liquid water, but we will show (in Section 2.7) that chains of water molecules with aligned effective dipoles can be stabilized.

Moreover, $V_{3}=-0.111$, which is quite close to $V_{1}=-0.116$. Since water molecules can easily be rotated, they are aligned according to this pattern inside very narrow pores [28]. They are then said to form wires or filaments. Perfectly linear polymerization with intramolecular exchange effects would yield

$$
\begin{aligned}
V_{5}= & 5 S(d, 0,0)+S\left(d, b_{1}, b_{1}\right)+S\left(d-1-a_{1}, b_{1}, 0\right)+S\left(d+1+a_{1}, 0, b_{1}\right) \\
& -2 S(d-1,0,0)-2 S(d+1,0,0)-2 S\left(d-a_{1}, b_{1}, 0\right)-2 S\left(d+a_{1}, 0, b_{1}\right) \\
= & -0.084
\end{aligned}
$$

An applied electric field does not only align water molecules, but also polarize water molecules in such a chain. This yields stronger bonds, since all positive charges of one molecule come closer to the central negative charge of the neighboring molecule. Polarization of water molecules is possible [29] and favors thus linear polarization by means of intramolecular exchange effects. 


\subsection{Initial Evidence of Molecular Chains}

When the young Theodor von Grotthuss was experimenting in 1806 with a Volta pile, he discovered that pure water has a much higher electric conductivity than other liquids. The chemical structure of water molecules was not yet known. [John Dalton asserted in 1808 that it is HO, while Avogadro proposed in 1811 that it could be $\mathrm{H}_{2} \mathrm{O}$. This hypothesis was disregarded, since it resulted from the assumption that all types of particles occupy the same volume in gases, whether they are molecules or atoms. This was only accepted at about 1860, after the development of the kinetic theory of gases.] However, Von Grotthuss knew that water molecules are composed of positive and negative parts, since they can be separated by electrolysis. This had already been proven in 1800 .

Von Grotthuss thought therefore that water molecules are held together in liquid water by mutual attraction of positive and negative parts. An electric field should align them. The high electric conductivity of liquid water could then be explained, if water molecules did contain tiny charge carriers that move more easily inside these chains than outside them [30]. This hypothesis may have been suggested by the method of fire-fighting of that time. People were standing in a row and passed buckets from hand to hand, but the existence of protons was not yet known. Nevertheless, this explanation was appealing and successful. Now, we can justify this model in term of intramolecular exchange effects. The left part of Figure 7 represents two water molecules in a linear chain, subjected to an electric field $\boldsymbol{E}$. One molecule contains an additional proton. We indicate displacements of charged particles by red lines, but the applied electric field does also modify the intermediate potential barrier. It becomes dissymmetric and facilitates "hoping" over the potential barrier for the proton in best position. The blue arrow represents a jump of this proton towards one of the nearby potential wells.

The right part of Figure 7 shows the result (without polarization effects). The $\mathrm{H}_{3} \mathrm{O}^{+}$ion became a normal $\mathrm{H}_{2} \mathrm{O}$ molecule, while the neighboring $\mathrm{H}_{2} \mathrm{O}$ molecule was converted into an $\mathrm{H}_{3} \mathrm{O}^{+}$ion. The proton did advance without being deviated by collisions. [Ohm's law is still valid for relatively small electric fields, as for ionic conduction in solid state physics]. We could equally well consider $\mathrm{H}_{2} \mathrm{O}$ molecules with one delocalized proton on the left side and an adjacent $\mathrm{HO}^{-}$ion. It contains only one proton, attracted toward the center of the next molecule. The electric field $\boldsymbol{E}$ would then cause a jump of the intermediate proton towards one of the two empty places. This would be equivalent to opposite motion of a proton hole. The essential result is that intramolecular exchange effects are realistic, since they explain the high electric conductivity of liquid water in a more detailed way.

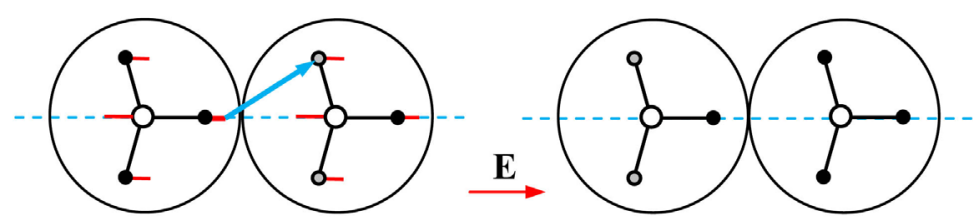

Figure 7. Explanation of the von Grotthuss mechanism. 


\subsection{Evidence of 2D Polymerization of Water Molecules}

The story of this discovery is similar to that of water memory. It began with an unexpected observation, made by the Russian chemist Nikolai Fedyakin. He condensed water in thin capillary quartz tubes and found that its physical properties are different from those of ordinary water. This phenomenon was totally unexpected, but arose at first much attention and scientific curiosity. Lippincot and Stromberg combined, for instance, routine infra-red spectroscopy with an improved method for producing this type of water. They confirmed that it has peculiar properties and proposed an explanation [31]. They assumed that water molecules can be bound to one another inside layers like that of Figure 8. The nature of hydrogen bonds was misunderstood (see Section 2.4), but this configuration was simple and heuristically useful. Since the layers can slide on one another, water remains liquid, but is viscous near substrates that favor this configuration.

2D polymerization of water molecules would thus yield a "new state" of liquid water, since it is partially crystallized. However, other persons declared that this is impossible, since they preferred to stick to customary ideas. The media propagated the slogan of "bad science", which had great impact. It led even to total prohibition of research on "polywater". Academic careers would have been broken for anyone who might dare to be involved in such "pathological" science. Even Stromberg, interviewed some 40 years later, accepted that researchers might be misled by unconscious bias [32]. However, he added that "most mistaken hypotheses in science aren't entirely wrong; they just have to be modified a bit."

Actually, it is well-known in material science that crystallization can be influenced by the substrate, because of local attractions. Rostrum Ray proposed that this phenomenon of epitaxy might explain water memory, because of the "extreme structural flexibility" of water molecules [33]. Their internal structure and relative positions could be modified, but this model would require molding and stability of detached structures and even multiplication of positive and negative molds in the course of successive EHDs. These hypotheses are not plausible enough, but Ray tried at least to explain water memory, instead of simply pretending that it is impossible.

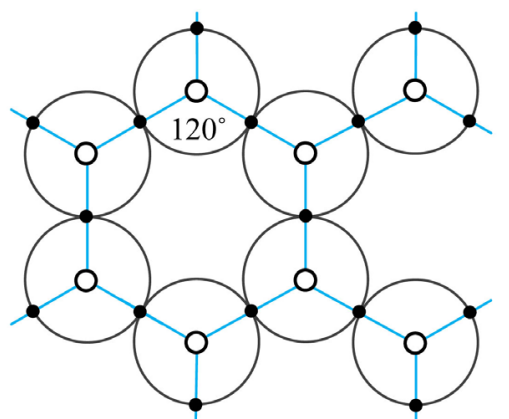

Figure 8. Polywater with hexagonal cells. 
$2 \mathrm{D}$ polymerization of water molecules was rediscovered by the bioengineer Gerald Pollack. He wondered why particles that imitate red blood cells can easily move through narrow capillaries. Trying to understand this fact, he realized that some materials create an "exclusion zone" near their surface [34]. Indeed, water molecules can be so strongly bound to the substrate and to one another in successive layers that the presence of red blood cells, for instance, becomes there impossible. Pollack adopted the model of Figure 8 to explain the formation of $2 \mathrm{D}$ lattices. He deduced from this model that every oxygen atom is surrounded by 3 half-hydrogen atoms. In natural units, this would yield a charge $(3 / 2)-2=$ $-1 / 2$ per molecule. Exclusion zones should thus be electrically charged and this was proven to be true. Contact of pure water with some materials is sufficient to constitute a battery.

Pollack's discovery and empirical investigations were outstanding achievements, but Figure 8 can be replaced by Figure 9. Delocalized protons have then 3 equally probable positions inside any water molecule. This model combines intramolecular with intermolecular exchange effects. It is then not necessary to assume strong distortions of tripoles in all water molecules, since the normal angles of $106^{\circ}$ can be preserved when the third angle is $148^{\circ}$. Regular hexagons would merely be transformed into elongated ones. The upper and lower rows of Figure 9 are even zigzagging chains, like those of Figure 6.

\subsection{Formation and Stabilization of Molecular Chains}

In interstellar space, there are ions that attract water molecules and align their effective dipoles, since the configuration $V_{2}$ of Figure 6 is sufficient for moderate electric fields. Figure 10 represents such a molecular chain in $3 \mathrm{D}$. The tripoles are alternatively orthogonal to one another and the aligned effective dipoles are represented by red arrows. On the average, the chain is axially symmetric. In outer space, ions would thus collect water molecules and become radially "haired". This does facilitate the participation of water molecules in the formation of planetary systems.

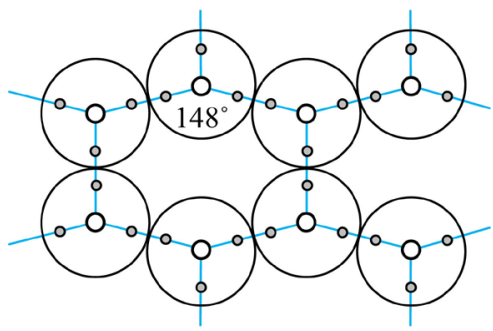

Figure 9. Polywater with elongated cells.

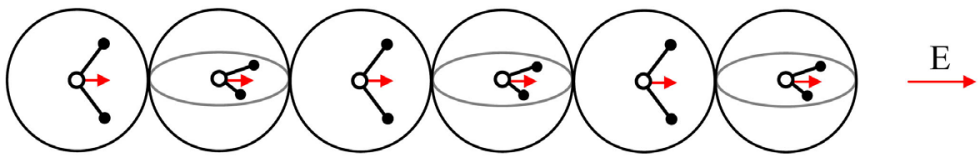

Figure 10. A single chain of water molecules. 
Although positive and negative ions are strongly bound to one another in ionic crystals, they are easily dissolved in liquid water, since the small water molecules are more attracted. They penetrate inside ionic crystals and dissolve them. In liquid water, ions will thus usually be isolated and surrounded by a "hydration sphere", where the effective dipoles of water molecules are oriented towards the central charge. This polarization decreases further away, because of thermal agitation. However, molecular chains could also be formed and rapidly stabilized by attracting one another. Figure 11 represents two possibilities by means of three water molecules that belong to parallel chains.

According to the dipole approximation (1), the electric field is $-p / r^{3}$, when $\theta=$ $90^{\circ}$. Real dipoles would thus be antiparallel in lateral positions, but we have to consider chains of tripoles. Two parallel molecular chains will thus be shifted by the distance $a$ with respect to one another. This allows for parallel or antiparallel effective dipoles. We expect that parallel ones are preferred, since the two protons of the lower molecule are then closer to the negative center of the upper right molecule. However, it is useful to verify if this leads to significant differences for the resulting potential energies $V_{a}$ and $V_{b}$. Since the potential energy of the upper pair is $V_{2}=-0.093$ in natural units, we get

$$
\begin{aligned}
V_{a}= & V_{2}+4 S\left(a, 0, d_{a}\right)+2 S\left(a, b, d_{a} \pm b\right)+4 S\left(D-a, 0, d_{a}\right) \\
& +2 S\left(d_{+} a, 0, d_{a}\right)+2 S\left(d_{+} a, 2 b, d_{a}\right)-2 S\left(0,0, d_{a} \pm b\right) \\
& -4 S\left(2 a, b, d_{a}\right)-4 S\left(d, b, d_{a}\right)-4 S\left(d-2 a, b, d_{a}\right) \\
= & -0.28 \\
V_{b}= & V_{2}+4 S\left(a, 0, d_{a}\right)+2 S\left(a, b, d_{a} \pm b\right)+4 S\left(d-a, 0, d_{a}\right) \\
& +2 S\left(d+a, 2 b, d_{a}\right)+2 S\left(d+a, 0, d_{a}\right)-2 S\left(0,0, d_{a} \pm b\right) \\
& -4 S\left(2 a, b, d_{a}\right)-8 S\left(d, b, d_{a}\right) \\
= & -0.09
\end{aligned}
$$

The \pm signs mean here that we have to sum two different terms. It appears that $V_{a}$ is 3 times lower than $V_{b}$. Agglomerations of parallel chains of water molecules are thus preferred. Moreover, the effective dipoles are already oriented in nearly the same way by the electric field of the ion. Molecular chains with parallel effective dipoles get spontaneously assembled and stabilized.

It is very important to realize that biologically active molecules contain electrically charged parts. They explain why these molecules are easily dissolved in liquid water and require at least contact with saliva. Figure 12 shows some typical examples. We see that the charged parts are even situated on a branch, where
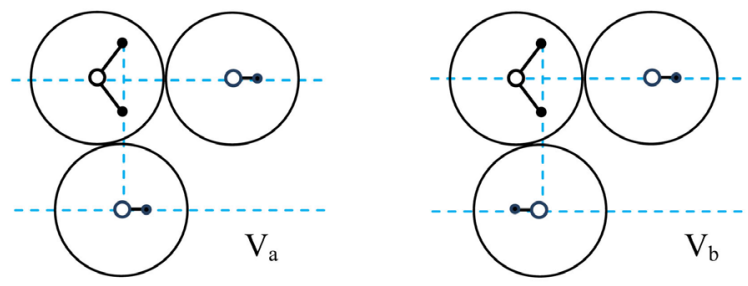

Figure 11. Mutual attraction of molecules on parallel chains. 


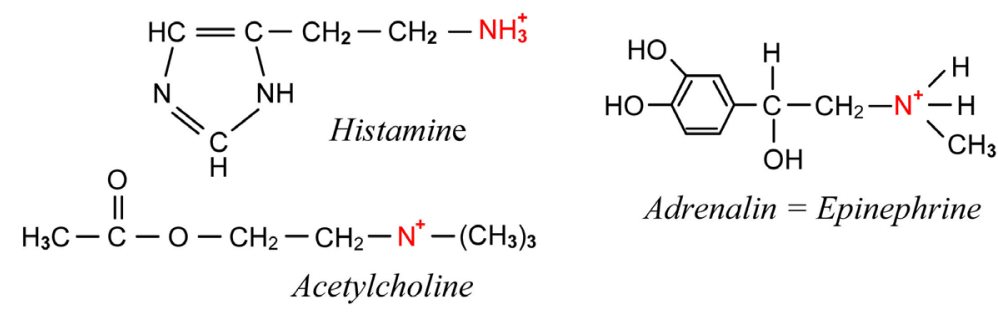

Figure 12. Biologically active molecules contain charged parts that can vibrate.

they can be set in oscillation by thermal agitation of water molecules in the surrounding liquid. They resonate at a particular frequency, which is characteristic of the active molecule. Its value is much lower than for vibrations of strongly bound charged particles inside molecules of any type. For active molecules, the resonance frequency is determined by the effective mass of its charged part and a weak restoring force. It results from deformations of the soft cocoon of polarized water molecules. These ideas are essential to unravel the puzzle of water memory.

\subsection{Single-Domain Ferroelectric Crystallites}

When the electric field of a biologically active molecule has started to assemble water molecules, it becomes a germ of ongoing crystallization. More and more water molecules are attracted and align their effective dipoles. This yields closely packed molecular chains, like that of Figure 10. A frontal view of the alternatively orthogonal tripoles yields a square lattice. Ideal alignment of all effective dipoles is only achieved in "single-domain ferroelectric crystallites". Water molecules are there more closely packed than in the surrounding water. We will prove that these crystallites contain many water molecules and are thus spherical. We will call them water pearls (WPs) and prove that they have the same size. Since they contain a great number $N$ of water molecules, their equally oriented effective dipole moment $p$ yields a very great total dipole moment $P=N p$ for every WP. Each one of them creates thus an electric field, which is able to assemble other water molecules. This process creates an adjacent WP and even a spontaneously growing chain of WPs.

To discard unnecessary objections, we mention that the solid state physicist Kittel realized already in 1946 that molecules of magnetite $\left(\mathrm{Fe}_{3} \mathrm{O}_{4}\right)$ create single-domain ferromagnetic crystallites [35]. Since they are ideal magnets, their discovery led to important applications, like magnetic recording. Blackmore discovered in 1975 that some species of aquatic bacteria collect $\mathrm{Fe}_{3} \mathrm{O}_{4}$ or $\mathrm{Fe}_{3} \mathrm{~S}_{4}$ molecules. They are then spontaneously assembled and constitute single-domain ferromagnetic crystallites [36]. They form chains of about a dozen beads. Magnetotactic bacteria produce these chains to remain in deeper layers of shallow waters and are able to sense the orientation of their internal compass needles in the inclined geomagnetic field of the Earth. They developed this stratagem to survive, since the oxygen content would be too high for them near the surface. This required the formation of specialized genes [37]. 
To determine the radius $R$ of these ferroelectric crystallites is a tricky problem. It has been tackled for the most common ferroelectric material $\left(\mathrm{BaTiO}_{3}\right)$ by means of the theory of phase transitions [38]. Since this approach does not apply to water molecules in the liquid state, we adopt another one. It does simply result from a transposition of the method that is used in electrostatics to determine the polarization of a homogeneous spherical particle. Figure 13(a) represents such a WP in pale blue color. Because of its surface charge, it orients water molecules in the surrounding liquid, but only in a limited region. The polarization of the surrounding water molecules is there progressively decreasing because of thermal agitation. This region is represented in a different color.

We use polar coordinates $(r, \theta)$ with axial symmetry. On the average, the WP is electrically neutral, because of the closeness of the charges $\pm 2 q$ inside all water molecules. The internal surface charge density results from the charges $\pm 2 q$ at the extremities of every molecular chain. It occupies the surface $d^{2}$ in planes that are perpendicular to these chains, but the surface of the sphere is inclined. The intersected surface is thus increased and the internal surface charge is $\sigma_{i}(\theta)=$ $\left(2 q / d^{2}\right) \cos \theta$ at the positive side. The external surface charge density $\sigma_{e}(\theta)$ is lower, but proportional to the internal one. The total surface charge density is thus $\sigma(\theta)=\sigma_{o} \cos \theta$. Positive and negative surface charges on opposite sides of a WP create a homogeneous electric field $E_{i}$ inside this sphere, as if it were composed of many very thin condensers. The electrostatic potential inside the WP is therefore $\phi_{i}(r, \theta)=E_{i} r \cos \theta$.

Figure 13(b) defines the potential $\phi(r)=(\mathrm{Q} / \mathrm{r}) \exp \left(-r / \lambda_{o}\right)$ that would be produced outside the sphere by the total charge $Q=N 2 q$ of all effective positive poles of water molecules, if this charge were situated at the center of the sphere. The usual Coulomb potential $(\mathrm{Q} / \mathrm{r})$ is modified by screening effects, resulting from positive and negative charges in the polarized region of the liquid water. The radial decrease is characterized by the Debye length $\lambda_{o}$. Figure $13(c)$ defines the actual potential $\phi_{e}(r, \theta)$ in the external medium. It is due to the charges $\pm Q$ of all positive poles and negative poles, separated by the distance $a$. The external potential depends then on $\Delta r=(a / 2) \cos \theta$, since



(a)

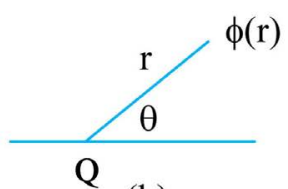

(b)

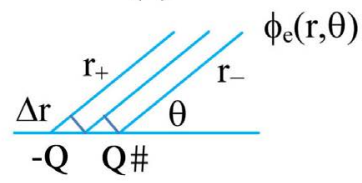

(c)

Figure 13. (a) A water pearl and the external domain of oriented water molecules; (b) A single charge $Q$ situated at the center of the sphere would generate an electric potential $\phi(r)$. (c) The actual external potential $\phi_{e}(r, \theta)$ results from two charges $\pm Q$. 


$$
\phi_{e}(r, \theta)=\phi(r-\Delta r)-\phi(r+\Delta r) \text { for } r \geq R
$$

$\phi_{i}(\mathrm{r}, \theta)$ and $\phi_{e}(\mathrm{r}, \theta)$ are subjected to boundary conditions, which determine the values of $R$ and $E_{i}$. This more technical problem is solved in the short appendix, but all required physical concepts have been explained here and the result is that $R \approx 10 \lambda_{o}$. The value of the Debye length $\lambda_{o}$ depends on the concentration of ions in the surrounding water. It is also proportional to the square root of the absolute temperature $\mathrm{T}$, but this factor is nearly constant between 20 and $30^{\circ} \mathrm{C}$. The value of $\lambda_{o}$ has been measured at $25^{\circ} \mathrm{C}$ for water with different concentrations of dissolved $\mathrm{NaCl}$ [39]. It appeared that $\lambda_{o} \approx 1 \mathrm{~nm}$ at $0.1 \mathrm{M}$ (mol/liter), but increases for lower and higher concentrations. Actually, $\lambda_{o} \approx 3 \mathrm{~nm}$ at $0.01 \mathrm{M}$ and $5 \mathrm{M}$, which is only slightly higher than for Dead Sea water. For pure water, $\lambda_{o}$ would depend on the concentration of $\mathrm{H}^{+}$and $\mathrm{OH}^{-}$ions. The radius $R$ of WPs is then somewhat smaller than $10 \mathrm{~nm}$.

\subsection{Properties of WPs and Their Poles}

When the particle physicist Shui-Yin Lo was visiting professor at the famous California Institute of Technology in 1996, he adopted a research project concerning properties of liquid water. He was surprised to discover that EHDs of $\mathrm{HCl}, \mathrm{NaOH}$ or $\mathrm{HNO}_{3}$ molecules in very pure water led to the formation of "novel stable structures". Lo thought that they result from crystallization of hydration spheres [40], while we attribute their existence to the formation of ferroelectric crystallites. This explains the dipolar nature of these particles and their great stability. They subsisted when all ions had been removed by successive dilutions and these structures were even multiplied in the course of successive dilutions, but only when they were followed by vigorous agitation.

When S. Y. Lo determined the sizes of various types of structures by means self-interference of scattered laser light, he found 3 distinct groups. The smallest particles had a diameter $D \approx 15 \mathrm{~nm}$ with very low dispersion. We interpret this result as meaning that $D$ is the diameter of WPs in pure water. Thus, $R \approx 7.5 \mathrm{~nm}$ and the Debye length $\lambda_{o} \approx 0.75 \mathrm{~nm}$. Since the volume occupied by every water molecule is $\mathrm{d}^{3}$, where $\mathrm{d}=0.275 \mathrm{~nm}$, WPs contain $N \approx 85,000$ molecules. This huge number justifies the assumption that they are spherical. Nevertheless, WPs are nanoparticles, since water molecules are very small.

The second group of structures, discovered by S.Y. Lo, had a size of about 300 $\mathrm{nm}$. We consider that this group corresponds to the length $L=N D$ of chains of WPs, containing $N \approx 20$ water pearls. We will explain (in Section 3.3), why their length $\mathrm{L}$ has to be limited. Its value depends on the mutual attraction between positive and negative surface charges on adjacent hemispheres. It is thus useful to replace the distributed surface charges of WPs by point-like poles. They are situated inside the sphere, like those of magnetized steel balls, but we can be more explicit. The total charge $Q_{o}$ on the surface of the positive hemisphere, is the integral of $2 \pi r \sigma(\theta) r \mathrm{~d} \theta$, where $r=R \sin (\theta)$ and $\sigma(\theta)=\sigma_{o} \cos \theta$, while the angle $\theta$ varies from $-\pi / 2$ to $+\pi / 2$. This yields $Q_{o}=(4 \pi / 3) \mathrm{R}^{2} \sigma_{o}$. We can also calculate the 
electrostatic potential $\mathrm{V}(\mathrm{x})$ for a test charge +1 that is situated on the symmetry axis at the distance $x$ from the center $\mathrm{O}$ of a WP. Figure 14 shows that $\mathrm{V}(\mathrm{x})$ is the integral of $2 \pi \sigma(\theta) r^{2} \mathrm{~d} \theta$ divided by the distance $R^{\prime}$. This allows us to define the effective charge $\mathrm{Q}(\mathrm{x})$ of the positive pole, if it were situated at the distance $R / 2$ from the center $\mathrm{O}$. This pole is represented by a black dot and

$$
V(x)=\frac{3 Q_{o}}{2} \int_{-\pi / 2}^{+\pi / 2} \frac{\sin ^{2} \theta \cos \theta \mathrm{d} \theta}{\left[R^{2}+x^{2}-2 R x \cos (\theta)\right]^{0.5}}=\frac{Q(x)}{x-(R / 2)}
$$

The result of numerical integration is shown in Figure 15. It appears that $\mathrm{Q}(\mathrm{x})=Q_{o}$ when $x>3 R$, but when the test charge is close to the surface of the $\mathrm{WP}$, it does mainly interact with the nearest surface charges. This reduces the value of $\mathrm{Q}(\mathrm{x})$. On the surface, $\mathrm{Q}(\mathrm{R})=0.68 Q_{o}$. We neglected all screening effects, but it is only important that neighboring poles are separated by the same distance $R$ and carry charges $\pm Q$.

\section{The Mechanism of Water Memory}

\subsection{Small Oscillatory Rotations of Water Pearls}

Because of the rapidly decreasing Coulomb forces, it is sufficient to consider the mutual attraction of neighboring positive and negative poles. At rest, they are aligned and their poles are separated everywhere by the same distance $R$, but small oscillatory rotations of WPs around their center will lead to transverse displacements of the poles. They are represented in Figure 16 by red arrows. For clarity, we exaggerated their magnitude. The essential point is, indeed, that neighboring poles remain in tangential contact and that the transverse displacements of the poles can vary along the chain. We characterize the instantaneous rotation of the $\mathrm{n}^{\text {th }}$ water pearl by the variable $u_{n}(t)$.

Figure 17 shows that when $\mathrm{u}$ is the relative displacement of two neighboring poles, they attract one another by the force $F$. In natural units, its magnitude is

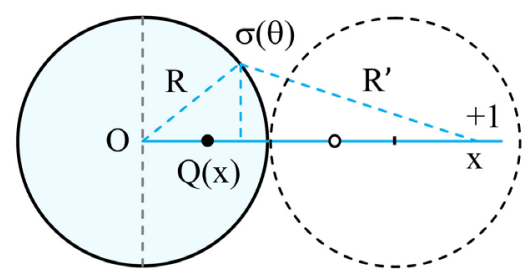

Figure 14. Definition of the charge $Q(x)$

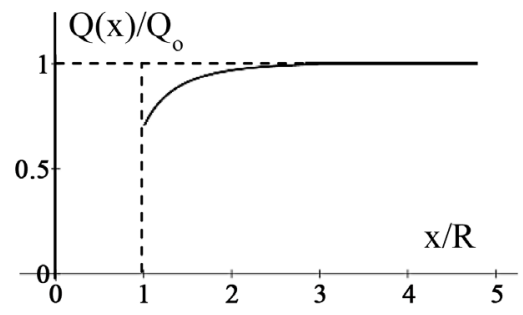

Figure 15. Calculated value of $Q(x)$. 


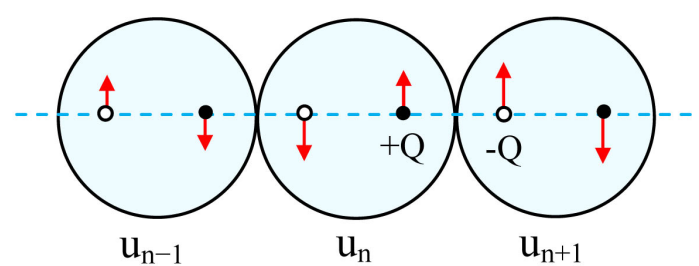

Figure 16. Small rotations of water pearls in a chain are specified by displacements of poles.

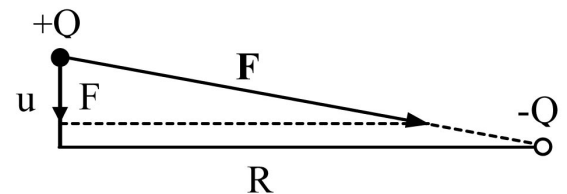

Figure 17. The restoring force is the transverse component of the force $F$.

$Q^{2} /\left(u^{2}+R^{2}\right)$. The transverse component $F$ is reduced by the factor $u / R$. It follows that when $u \ll R$, the restoring force is

$$
F(u)=-K u \text { where } K=\frac{Q^{2}}{R^{3}}
$$

This force is proportional to the relative displacement $u$, as for any elastic system. When $M$ is the effective inertial mass of poles, the equation of motion for the $\mathrm{n}^{\text {th }}$ water pearl is

$$
M \ddot{u}_{n}=K\left(u_{n+1}-u_{n}\right)-K\left(u_{n}-u_{n-1}\right)
$$

Every dot stands for a time derivative. Since this equation is identical for all WPs, it describes the behavior of the whole chain. It can be simplified when the displacements $u_{n}$ are smoothly varying along the chain, which is equivalent to saying that the diameter $D$ of WPs is small compared to the distance where the relative displacements $u_{n}$ are notably varying. We can then replace $u_{n}(t)$ by $u(x$, $t$ ), where the coordinate $x$ is treated as if it were a continuous variable. Actually, $u_{n \pm 1}=u_{n} \pm D u^{\prime}+D^{2} u^{\prime \prime} / 2$, where $u^{\prime}$ and $u^{\prime \prime}$ designate first and second order partial derivatives with respect to $x$. Equation (6) is then reduced to

$$
\ddot{u}=v^{2} u^{\prime \prime} \text { where } v^{2}=D^{2} K / M
$$

This is the usual wave equation for vibrating strings. An infinite chain would allow for $u(x, t)=u(x \pm v t)$. This corresponds to a function of any shape, moving at the velocity $v$ towards the right or the left. Possible attenuations of oscillatory rotations have been neglected in (6) and (7), but will be discussed later on.

\subsection{Standing Waves on Chains of Water Pearls}

For a chain of finite length $L$, we have to know the boundary conditions at $x=0$ and $x=L$. When both ends are free, the first and last pearls are not subjected to any force. Thus, $u^{\prime}(x, t)=0$ for $x=0$ and $x=L$. In other words, $u(x, t)$ has to reach maximal values at both extremities. This allows for a particular solution of well-defined frequency $f$ and well-defined wavelength $\lambda$ : 


$$
u(x, t)=A \cos (k x) \sin (\omega t) \text { where } \omega=2 \pi f \text { and } k=2 \pi / \lambda
$$

Since $u^{\prime}(x, t)$ is proportional to $\sin (k x)$, the boundary condition $u^{\prime}(0, t)=0$ is satisfied, but $u^{\prime}(L, t)=0$ requires that $k L=s \pi$, where $s=1,2,3, \cdots$. It follows that $L=s \lambda / 2$ and because of (7), that the spectrum of possible frequencies is defined by

$$
f=\frac{v}{\lambda}=s \frac{v}{2 L}=f_{s} \text { where } s=1,2,3, \cdots
$$

The only possible frequencies are thus integer multiples of the fundamental frequency $f_{1}=v / 2 L$. For sound waves, any pair of such frequencies would produce an impression of harmony. The spectrum $f_{s}=s f_{1}$ is therefore said to be a "harmonic" one. These properties are well-known in physics, but everyone should see why a chain of WPs with free ends does only allow for standing waves. This means that for any particular solution (8), all WPs are set in oscillatory rotations at the same frequency $f$, but the amplitude of these oscillations varies along the chain.

However, the approximation (7) is of limited validity, since it requires that $D \ll L$. The measurements of Lo imply that chains of WPs contain a relatively small number of WPs $(N \approx 20)$. To see how far the approximation $(9)$ is realistic, we have to solve the general equation (6). This is easy when we use complex notations, since standing waves are then defined by

$$
u_{n}(t)=A \mathrm{e}^{i\left(k x_{n}-\omega t\right)} \text { where } \omega^{2}=\frac{K}{M}\left(\mathrm{e}^{i k D}+\mathrm{e}^{-i k D}-2\right)
$$

Thus,

$$
\omega(k)=v \frac{\sin (k D / 2)}{D / 2} \text { and } f(\lambda, D)=\frac{v}{\pi D} \sin (\pi D / \lambda)
$$

The function $f(\lambda, D)$ is represented by the dark curve in Figure 18. It reveals that the domain of possible frequencies is limited, as well as the domain of possible wavelength. It is only physically possible that $\lambda / 2 \geq D$. The approximation $f$ $=v / \lambda$ corresponds to the red line, which would allow for arbitrarily high frequencies, but (10) imposes an upper limit $\left(f_{\max }=v / \pi D\right)$. Nevertheless, the linear approximation is valid for a relatively large domain of low frequencies. [Indeed, $\sin (x)=x-x^{3} / 6 \approx x$, when $\left.x \ll 2.5\right]$. Actually, the spectrum of possible frequencies is $f_{s}=s f_{1}$ when $s \ll N$.

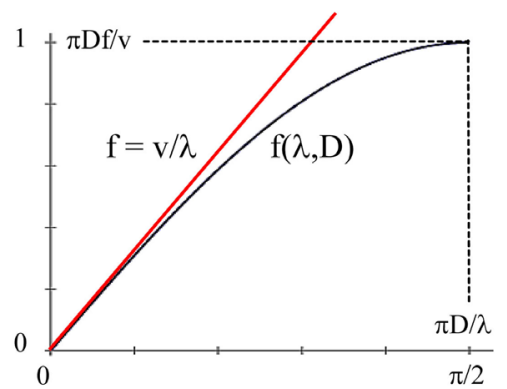

Figure 18. The general function $f(\lambda)$ for chains of WPs. 


\subsection{Automatic Trimming of Chains of Water Pearls}

Figure 19 summarizes the essential steps that explain why chains of water pearls are the information carriers of water memory. The upper line represents a biologically active molecule, where the charged part oscillates at some very low frequency $f$. It cannot communicate these oscillations to the WPs, since the length of the chain $L<\lambda / 2$, where the wavelength $\lambda$ is determined by $f=v / \lambda$. The second line represents what happens as soon as the length of the chain of WPs reaches the value $L=\lambda / 2=v / 2 f$. The electric field of the oscillating charge does then excite a standing wave for oscillatory rotations of WPs.

The last WP of the growing chain does suddenly start to oscillate when its length $L=v / 2 f$. The amplitude of this oscillation is the same as for the first WP of the chain, which can also be set in forced oscillation by the active molecule. It remains attached to it, but can now communicate its motion to other WPs of the chain. However, the rotation of the last pearl of the chain prevents the formation and attachment of an additional WP. The growing chain is thus automatically trimmed. Information that is characteristic of the type of active molecules has been encoded by means of the length $L$ of the chain. It depends indeed on the frequency $f$.

The third line of Figure 19 shows that when the trimmed chain is detached from its generator, it does still allow for a standing wave for the same length $L=$ $\lambda / 2$. The liberated chain of WPs conserves the acquired information. Moreover, it produces itself an oscillating electric field. Its frequency $f$ is adequate to create more equally trimmed chains of WPs. They are thus multiplied by an autocatalytic process. This provides the key for a rational justification of water memory. It resulted from a systematic examination of all possible interactions between water molecules and their logical consequences.

We might object that oscillatory rotations of WPs will be damped by friction, exerted by surrounding water molecules. This is true, but trimmed chains are also subjected to local impacts of water molecules. Although the impacts are random, the chain does pick-up energy when it allows for resonances at any frequency $f_{s}$ for possible standing waves. Since it is sufficient that the free ends of the chain can oscillate with maximal amplitude, standing waves of smaller wavelengths and higher frequencies can also be excited. Excitation of a standing wave at a higher frequency can easily be demonstrated with a flute, since "overblowing" is sufficient to double the frequency for standing waves, without modifying the effective length for longitudinal oscillations. Oscillatory rotations of WPs at

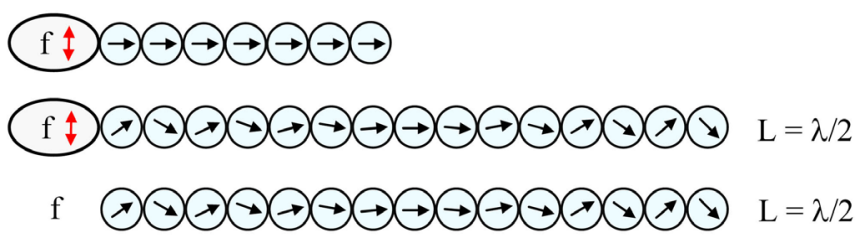

Figure 19. Creation of trimmed chains of water pearls, resonating at the frequency $f$ of the active molecules for chains of length $L$. This remains true for detached chains. 
higher frequencies imply more rapid motions and thus greater kinetic energies and more violent local impacts. Available energies depend on the statistical distribution of kinetic energies of water molecules in the liquid state. Although a chain of WPs of given length allows for a superposition of different modes of oscillations, those of increasingly higher frequencies will thus be excited with decreasing amplitudes. They are byproducts of random re-excitation, but the lowest possible frequency $f_{1}$ remains the predominant one.

\subsection{Negation of Water Memory Was Based on False Assumptions}

Benveniste's experimental proof of water memory was categorically rejected because of prevailing beliefs. They resulted from four erroneous assumptions:

1) Biologically active molecule can have no effects any more, when all of them have been eliminated by successive dilutions.

2) Even if biologically active molecules could create substitutes, made of water molecules, they would have to be adaptable. Such aggregates are unknown and can thus not exist.

3) Biologically active molecules can only act on their specific receptors by means of chemical affinities. Local structuring of liquid water would be unable to mimic these processes. This is particularly implausible for various types of molecules, since that would require an extraordinary capacity of adaptable imitation.

4) Extra high dilutions are also used for homeopathy, which is inefficient. The preparation of EHDs does even involve shaking by vigorous "successions". This ritual is a sign of charlatanism.

We have already shown that the two first objections are contradicted by the formation of trimmed chains of WPs. The third objection concerns the fundamental problem of molecular interactions. Modifications of the state of motion can result from direct contact (collisions), but also from actions at a distance (due to attractive or repulsive forces). We are accustomed to the idea that structural changes (combinations or dissociations) at molecular level result from chemical reactions, requiring direct contact, chemical affinities and configurational conformity. However, internal modifications can also result from energy transfer (excitation or disexcitation) by means of force fields.

Figure 20 summarizes the required restructuring of our ideas, because of the concept of ferroelectric water pearls. First of all, we have to realize that biologically active molecules contain an electrically charged part (Figure 12) that has a resonance frequency $f$. It creates thus an electric field that oscillates at this frequency. The upper line of Figure 20 represents the normal process, where this electric field acts on a molecular receptor, which has also a charged part that can oscillate. Specific receptors of a particular type of active molecules contain also a charged part that can oscillate. It resonates at a frequency $f_{r}$ It is thus sufficient that $f_{r} \approx f$ to allow active molecules to stimulate their specific receptors. There is some tolerance, since the probability of interaction by resonance corresponds to a peak that has some width. 


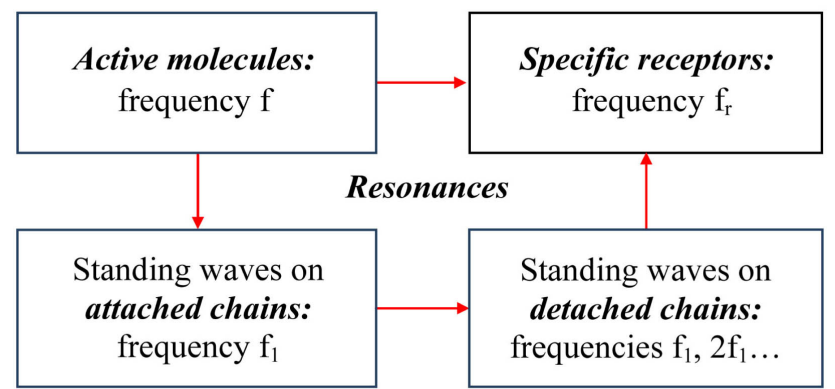

Figure 20. Water memory reveals a new type of molecular interactions.

The assumption that molecular interactions are only possible according to the "key and slot model" of chemical reactions is not correct. Biologically active molecules can also interact with their specific receptors by means of oscillating electric fields and resonances. This allow for a bypass, represented by the second line of Figure 20, since the oscillating electric field of active molecules can also create trimmed chains of WPs. As long as they are attached to their generators, they resonate at a frequency $f_{1} \approx f$. This remains true when these chains are detached, but reactivation of their oscillations by thermal agitation in liquid water leads to a harmonic spectrum of possible frequencies $\left(f_{s}=s f_{1}\right.$, where $s=1,2,3, \cdots)$. The fundamental frequency $f_{1}$ remains dominant, however. Standing waves on trimmed chains of WPs do produce an oscillating electric field of frequency $f_{1} \approx f_{r}$ and can thus stimulate the same receptors.

The collective electric field, generated by all trimmed chains of WPs, has even the capacity to create more and more equally trimmed chains. Their number is increased and the oscillating electric field is amplified by an autocatalytic process. The possibility that molecular interactions can result from oscillating electric fields and resonance effects had been overlooked. The discovery of water memory did thus reveal the existence of a mechanism that is of fundamental importance and even very efficient.

The fourth erroneous assumption concerns homeopathy. Since the underlying mechanism was not understood, it was believed that its efficiency can only result from placebo effects. We wonder how they can be justified for animals and small children. Our purpose is not to defend homeopathy, but to restore truth, also in this regard. It is therefore instructive to examine the argumentation advanced by those who would like to eliminate homeopathy. The Australian National Health and Medical Research Council published in 2015 a study on "Evidence on the effectiveness of homeopathy" [41]. This report was sponsored by the Australian Government, but was not based on a scientific study of underlying physical and physiological process. It was merely a collection of 176 articles, supporting the claim that "there are no health conditions for which there is reliable evidence that homeopathy is effective."

These evaluations are essentially dependent on subjective appreciations. It was recognized that the general conclusion of their report was "based on all the evidence considered". Other evidence was discarded. The first report of 2012 had 
even been concealed [42], since it was not sufficiently selective in this regard. The published report stated that the aim of this study was "to assist people in making health care choices", but it was addressed to political deciders. The authors mentioned, indeed, that they wanted to "influence policy" and to get "increased funding for such research" ([41], pp. 6, 16 and 4). This report was widely publicized and is equivalent to lobbying.

\subsection{The Standard Procedure for Extra-High Dilutions}

It is even necessary to clarify the origin of homeopathy, which has often been misrepresented to denigrate it. The basic idea was due to Samuel Hahnemann (1755-1843). He was a regular medical doctor. After acquiring his diploma at the age of 24 , he practiced during 5 years, but decided then to cease. He had realized, indeed, that it would have been better for some of his patients not to be treated according to the "art of healing" of his time. He was even horrified that he might "murder" suffering people, instead of helping them. This was an exceptionally honest attitude, justified by recognizing the cause of this horrible situation. Neither the chosen substances, nor the doses were determined in a rational way, although Paracelsus wrote already in 1543 that "only the right dose differentiates a poison from a remedy".

Hahnemann's linguistic gifts made it preferable for him to translate books and to search there for possible improvements of medical practice. In one of these books, it was claimed that the bark of a Peruvian tree was able to treat malaria. It is known today that the bark of "cinchona" trees contains quinine. Most efficient medicines were actually discovered by trial and error. It was already known in Antiquity, for instance, that leaves of willow trees can stop pain. A chemist discovered in 1853 that the active molecule is $\mathrm{C}_{9} \mathrm{H}_{8} \mathrm{O}_{4}$, which became famous as aspirin. Even elephants, apes and other animals know how to cure or avoid ailments [43]. The textbook attributed the beneficial effects of cinchona powder to its taste, but Dr. Hahnemann could not believe this claim. Nevertheless, he decided to verify if there were any detectable effects and was amazed that it produced malaria-like symptoms.

By experimenting with other substances, he realized that medicines could be discovered in a more rational way, by adopting the "law of similars". This was merely an empirical rule, but such rules were often followed before understanding why they are valid. [Even Newton's law of gravity was expressed in terms of actions at a distance. It did account for observed phenomena, but the real cause is a gravitational field, which corresponds even to modifications of the metric of space and time.] Since Hahnemann tried to discover medications by means of tests, performed on healthy persons, he had to use the lowest possible doses. $\mathrm{He}$ adopted thus the method of successive dilutions. If the result was beneficial, such an EHD could also be administered to patients in a secure way. We recall that Hahnemann was a learned medical doctor and was thus able to verify if a preparation is helpful of not. 
As an example, we mention Apis mellifica. Until recently, it was customary in medicine to use Latin, also for anatomy, to overcome language barriers. The European honey bee is called "Apis mellifera" and the main component of its venom is mellitine. This molecule has also anti-inflammatory properties and honey bees do even protect their larvae from infections by means of very efficient substances. Hahnemann presented his discovery already in 1796 in a German medical journal, by formulating the rule that "like cures like". Objections that are based on the finite divisibility matter are anachronistic. [The ancient concept of atoms had been reintroduced by Boyle in 1661 and elaborated by the chemist John Dalton in 1804, but the atomic theory was only accepted at about 1860, since the kinetic theory of gases did prove that Avogadro's hypothesis was correct. Nevertheless, Mendeleev did not yet dare to use the concept of atoms in 1869.]

Hahnemann could thus assume that even when a substance has been diluted many times, there remains something of this substance. In 1810, he presented a first collection of results and one year before his death, the $6^{\text {th }}$ edition of his "Organon of the Rational Art of Healing" was ready for publication. It is easily available [44]. It should also be obvious that successive dilutions required always homogenization before the next step. Hahnemann did this by holding the vessel in his hand and stroking it with vigor on a semi-elastic surface, like leather. Modern chemists use mechanical vortexing to insure homogenization of mixtures. This is merely a simpler method. We will show in the following section that these "successions" do also have another effect. Although Hahnemann was only concerned with practical medicine, he discovered already the bypass of Figure 20. Neither he nor his detractors were aware of this fact. Even when Benveniste did empirically prove the biological efficiency of EHDs, it was categorically declared to be impossible.

\subsection{The Quasi-Periodic Variations of Biological Efficiency}

Sir John Maddox, long-term editor of Nature (1966-73 and 1980-95) accused Benveniste of self-delusion, although his article contained two figures, displaying results of measurements [1]. Figure 21 reproduces one of them. It is undistorted

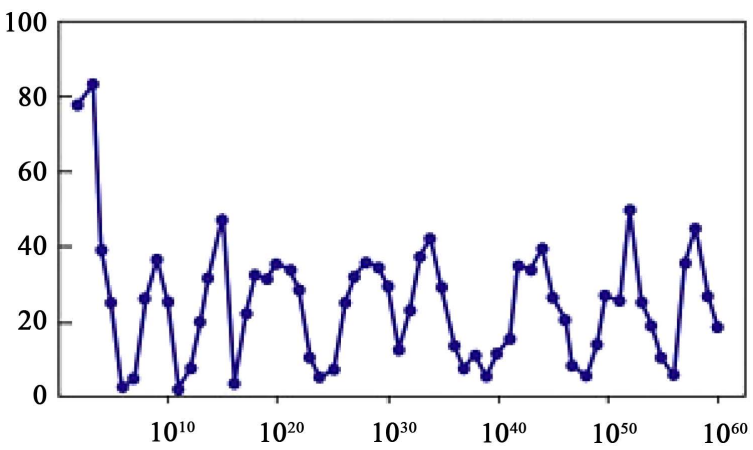

Figure 21. Measured quasi-periodic variations of the biological efficiency of EHDs, published by Benveniste et al. [1]. 
and was extracted from a publication in Japanese. We see 9 peaks. The first one is higher than the following ones. Since every peak did result from several measurements, the investigators should have realized that the quasi-periodic variations cannot result from "disregard of statistical principles" and "sampling errors". The investigators proclaimed even that Benveniste's experimental results were merely due to self-delusion [2]. What would result from the theory of water pearls? We begin with a description of the underlying processes in usual language by means of Figure 22. The first frame (a) shows some of the initially diluted active molecules, their charged parts (in red) and attached trimmed chains of WPs. We know that their length $L=\lambda / 2$ and depends on the frequency $f$ of the oscillating electric field of the active molecules. The second frame (b) illustrates the situation immediately after the first dilution and vigorous agitation. The concentration of active molecules has been reduced and agitation lasted long enough to detach all chains from the remaining ones, but some chains of WPs were broken.

The third frame (c) shows that after a relatively short time interval, the remaining active molecules had again formed trimmed chains of WPs. Broken detached chains did grow and new ones were generated by the global oscillating electric field. These chains have the same characteristic length $L$, allowing for standing waves at the frequency $f_{1}$ as well as harmonics $\left(2 f_{1}, 3 f_{1}, \cdots\right)$. Some chains may have reached the length $2 L$. It allows for a mode of oscillation where $2 L=\lambda$, which is equivalent to $L=\lambda / 2$ and allows for the frequency $f_{1}$. There did also appear some "associated chains", resulting from mutual attraction of trimmed chains. Figure 23 shows how two parallel chains of WPs will be attached to one another, but more than two chains are also possible. However, associated chains do not resonate at the same frequency or not at all.

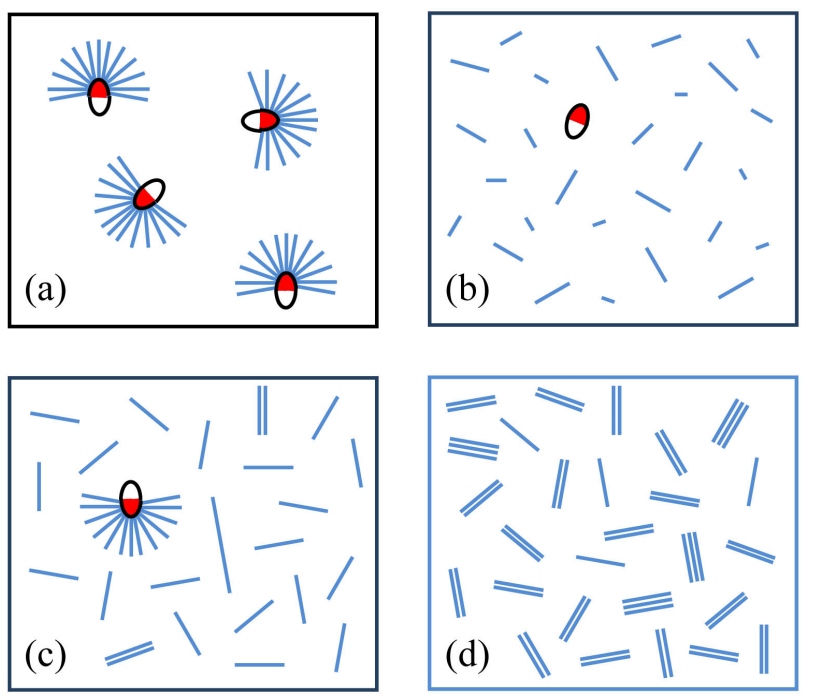

Figure 22. Decoding the standard procedure for preparing EHDs. (a) Active molecules and attached trimmed chains of WPs; (b) Detached and broken chains, immediately after dilution and agitation; (c) Reconstituted chains; (d) Excess of associated chains. 


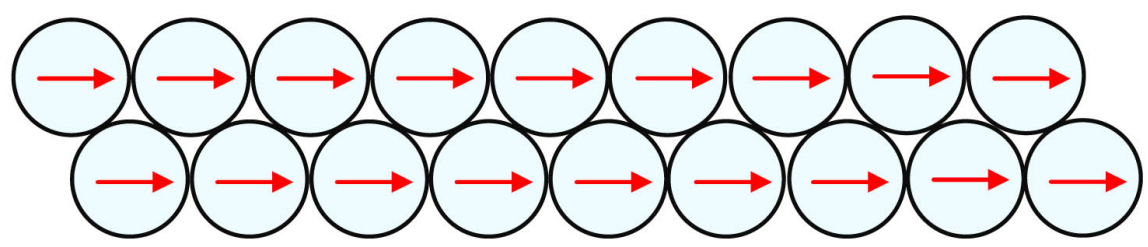

Figure 23. A simple associated chain of water pearls.

The fourth frame (d) of Figure 22 represents a state where nearly all single chains of WPs got bound to one another. The biological efficiency of the resulting EHD is then much reduced, but associated chains can be broken by vigorous agitation. Single chains can then be multiplied again during successive dilutions, until there are so much of them that association will be favored. The global result is that the biological efficiency of EHDs has to vary in a quasi-periodic way. It is not perfectly periodic, since these processes allow for statistical fluctuations.

\subsection{Kinetics of Water Memory}

It is useful to express these ideas by means of equations, since they allow for logical deductions. Let $X_{o}$ be the initial number of active molecules, dissolved in a given volume of twice distilled water. This concentration is reduced by successive dilutions, where the same fraction of the homogenized solution is eliminated at every step. Usually, this fraction is $9 / 10$ or $99 / 100$. It is replaced by pure water to get always the same volume. When successive dilutions follow one another at identical short time intervals $\Delta t$, the concentration of active molecules becomes a function $X(t)$ that decreases step-wise, since

$$
X(t+\Delta t)=X(t)-\alpha \Delta t X(t)
$$

The value of $\alpha \Delta t=0.9$ or 0.99 . For smalltime intervals, $X(t)$ can be treated as if it were a continuous function. It decreases then according to the equation:

$$
\dot{X}=-\alpha X \text { so that } X(t)=X_{o} \exp (-\alpha t)
$$

The exponential decrease does necessarily end up with $X(t)=0$ when $t \gg 1 / \alpha$, but this does not prove that the biological efficiency of EHDs has to vanish. Active molecules are able to generate trimmed chains of WPs with a probability $g$ per unit time and they do generate more of them with a probability $\beta$ par unit time. The concentration $Y(t)$ of trimmed chains of WPs increases thus according the equation:

$$
\dot{Y}=g X+\beta Y
$$

When the sequence of EHDs starts without previously formed trimmed chains, the initial value $Y(0)=0$. Because of (11) and (12), we get then

$$
Y(t)=\frac{A}{\alpha+\beta}\left[\mathrm{e}^{\beta t}-\mathrm{e}^{-\alpha t}\right] \text { where } A=g X_{o}
$$

It appears that $Y(t)=A t$ when $t \rightarrow 0$. The initial increase of $Y(t)$ is thus linear 
and very rapid when $A$ is great. When the generation of new substitutes exceeds losses $(\beta>0)$, the concentration $Y(t)$ does eventually increase like $\exp (\beta t)$. This constantly accelerated increase would only stop when the whole amount of liquid water has been solidified. This might even apply to oceans and would be catastrophic, but is prevented by forming associated chains. Their concentration $Z(t)$ varies also, but

$$
\begin{gathered}
\dot{Y}=A \exp (-\alpha t)+\beta Y-\varepsilon Z Y-\varepsilon^{\prime} Y^{2} \\
\dot{Z}=\varepsilon Z Y+\varepsilon^{\prime} Y^{2}-\gamma_{o} Z
\end{gathered}
$$

Equation (15) accounts for the fact that $Z(t)$ increases by combining already existing associated chains with single ones. The average rate $\varepsilon$ is greater than for association of two single chains, because of more possibilities. (12) is replaced by (14), since associations imply that $Y(t)$ decreases by the same amount. However, every associated chain has also a probability $\gamma_{o}$ per unit time to be destroyed by vigorous agitation. Figure 24 presents the results of numerical integration of (14) and (15). The unit of time is $\tau_{o}=1 / \alpha=1$. We assumed that $A=50$ and that $\beta=0.5, \varepsilon=0.1, \varepsilon^{\prime}=0, \gamma_{o}=1.5$. The measured efficiency is proportional to $Y(t)$. This function is thus represented by a thicker line.

The red line describes the exponential decrease of the concentration $X(t)$ of active molecules and the thin blue line represents the variations of the concentration $Z(t)$ of associated chains. It was a "hidden variable" for Benveniste and his team. The first peak of $Y(t)$ is greater than the other ones, since $X(t)$ does still contribute to the generation of substitutes. The initial increase is linear and very rapid when $A=g X_{o}$ is great. This accounts for Figure 21. The following peaks were not always identical, but they had nearly equal heights. They are perfectly periodic in Figure 24, since we assumed constant parameters in (14) and (15). We assumed that $Y(0)=0$ and $Z(0)=1$, since $\varepsilon^{\prime}=0$ requires that one of the initial values $Y(0)$ or $Z(0)$ is different from zero to initiate the periodic variations. It should be noted that the peaks for $Y(t)$ are dissymmetric. The increase is progressive, but the decrease is precipitated, since single chains are more frequently associated with already existing ones when their concentration $Z(t)$ is great. This was not obvious in Figure 21, but has been demonstrated by Montagnier (Section 4.1).

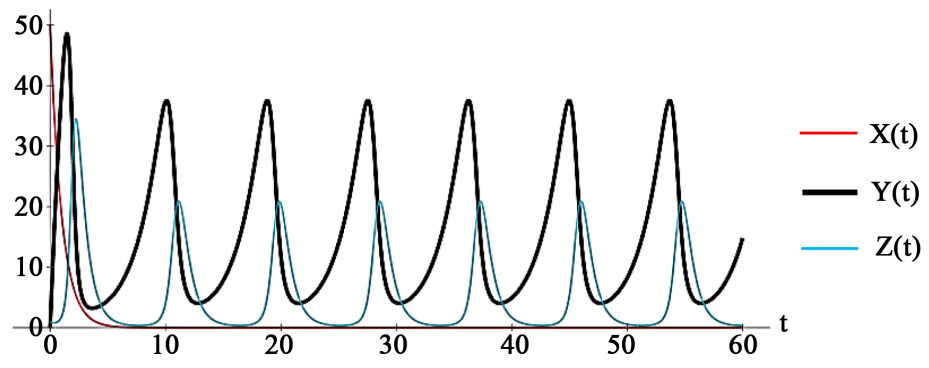

Figure 24. Decrease of the concentration $X(t)$ of active molecules and the periodic variations of the concentrations $Y(t)$ and $Z(t)$ of single and associated chains of WPs in the course of successive dilutions. 
It is simply a matter of fact that if Maddox had tried to understand the experimental results, he would have discovered that similar variations were already known since 1910 for autocatalytic reactions and so-called "chemical clocks". Periodic variations attracted even more attention in the $1920^{\text {th }}$, since they were also observed for variations of the population density of predators and their prey. Predators proliferate when pray is abundant, but when the population of victims has been decimated, the predators have greater difficulties for their own survival and reproduction. Fewer predators allow the population of potential victims to grow again. This phenomenon was described by the famous Lotka-Volterra equations, which are identical to (14) and (15), when $A=0$ and $\mathcal{\varepsilon}^{\prime}=0$.

After developing the present theory, we found the book of Francis Beauvais [10]. He provided many examples of similar results obtained by Benveniste and his team and referred even to the Lotka-Volterra equations. Without the concept of WPs, he had to assume the formation of negative and positive molds. This hypothesis was only mentioned as an example, but proves that Maddox could have referred to the Lotka-Voltera equations, if he had tried to understand the unexpected experimental results.

Thomas Kuhn analyzed the process of scientific revolutions [45]. He distinguished different stages. When "anomalies" appear and cannot be explained by means of "normal science", the first reaction is to declare that they are impossible. Confirmation of the reality of these anomalies opens a period of "crisis". Various types of approaches are then tried and momentarily permitted. [For instance, Michelson's experiments of 1881 and 1887 did prove that the velocity of light with respect to the Earth is identical for any direction. This was not compatible with the concept of light waves propagating in ether with respect to the moving Earth. It was proposed at first that the ether could be entrained by the Earth, but Einstein realized that no luminipherous ether is needed. This hypothesis can be dropped, indeed, when we accept that results of space and time measurements depend on the chosen reference frame. Only the velocity $c$ of light in vacuum has always the same value for any inertial frame.] This "paradigm shift" resulted thus from reexamining previous assumptions and correcting them. This is also necessary for water memory, because of the third assumption in Section 3.4.

\subsection{Properties of the Limit Cycle and Aging}

Consequences of (14) and (15) can also be expressed by displaying the variations of $Y(t)$ versus those of $Z(t)$. Any particular point $(Y, Z)$ defines then the state of the system at some instant $t$ and the evolution of this state is represented by a continuous line. The dark curve in Figure 25 is equivalent to Figure 24. The green curve results from identical parameters, but starts at $(0,10)$, instead of $(0$, $1)$. Since the presence of more associated chains does immediately reduce the concentration of single chains, the first peak of Figure 24 is reduced. However, 


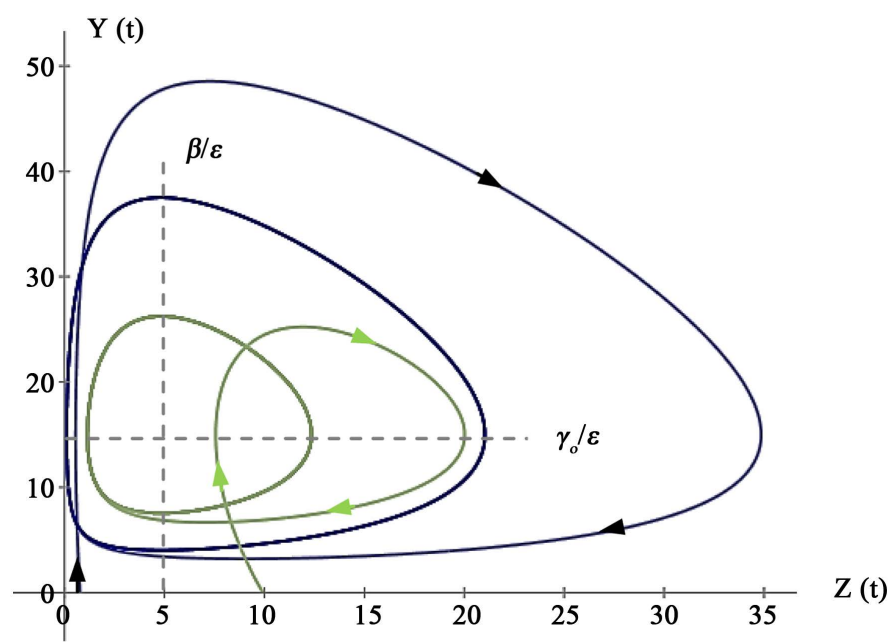

Figure 25. Parametric representation of the evolution of EHDs.

maximal and minimal values of $Y(t)$ are always reached when $Z=\beta / \varepsilon$. Those of $Z(t)$ require that $Y=\gamma_{d} / \varepsilon$. These facts result from (14) and (15) when $\dot{Y}=0$, $\dot{Z}=0$ and $\varepsilon^{\prime}=0$.

Figure 26 shows what would happen for $\varepsilon^{\prime}=0.0001$, when all other parameters have the same values as for Figure 24. We assumed again that $A=50$, but the first peak of $Y(t)$ is greater, since $Z(t)$ was immediately increased by $\varepsilon^{\prime} Y^{2}$. However, the amplitude of the periodic variations of $Y(t)$ is decreasing. The article of Benveniste [1] contained a second figure for a longer series of EHDs. It suggests a possible decrease of $Y(t)$, but $\mathcal{\varepsilon}^{\prime}$ was surely smaller than 0.0001 .

Equations (14) and (15) allow us also to answer two important questions concerning aging. Should the sequence of successive dilutions be stopped at an instant where the biological efficiency has a high value? Does the efficiency of homeopathic preparations not totally vanish after some time? To answer these questions, we solve Equations (14) and (15) after the instant $t=0$, where the process of EHDs was stopped. We assume that all active molecules were already eliminated $(A=0)$, but without shaking, the values of $\beta$ and $\gamma_{\mathrm{o}}$ are smaller. Figure 27 shows the results of numerical integrations when $\beta$ is reduced from 0.5 to 0.1 and $\gamma_{o}$ from 1.5 to 1 , while $\varepsilon$ and $\varepsilon^{\prime}$ are not modified. The black curve results from $Y(0)=30$ and $Z(0)=1$, while the red one would be due to $Y(0)=5$ and $Z(0)=10$. We see that the initial conditions are irrelevant for the final result, although we assumed that $\varepsilon^{\prime}=0.0001$.

It follows indeed from (14) and (15) that $Y(t) \rightarrow \gamma_{\mathrm{o}} / \mathcal{E}=10$ and $Z(t) \rightarrow \beta / \mathcal{E}=1$ for $\varepsilon^{\prime}=0$, when $\dot{Y}=0$ and $\dot{Z}=0$. The correction for small values of $\varepsilon^{\prime}$ can be obtained by introducing the lowest order approximation in the same equations. The reduction of the final values is negligible when $\varepsilon^{\prime}=0.0001$. It is remarkable that the alternative dominance of single and associated chains continues during the initial period of aging. We have also to stress the fact that preservation of the biological efficiency of EHDs requires that the system has not been perturbed. This can happen by heating and ultrasound, but also in another way. 


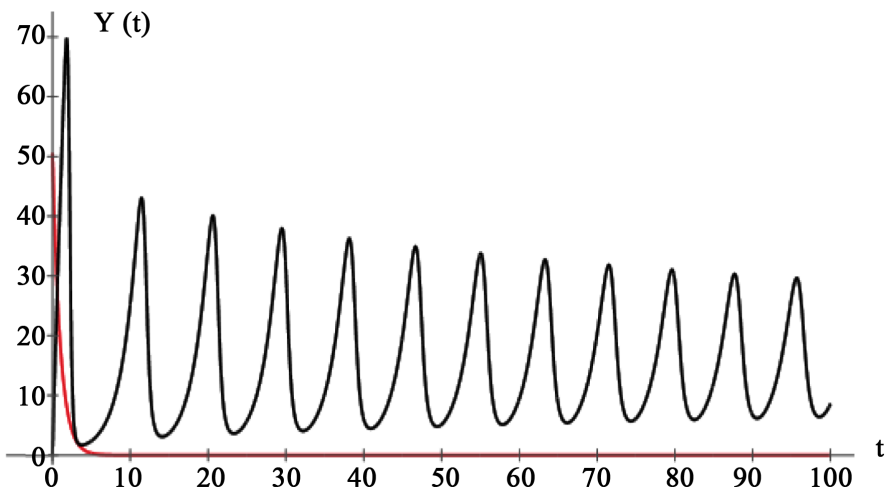

Figure 26. Possible variations of $Y(t)$ for many successive dilations.

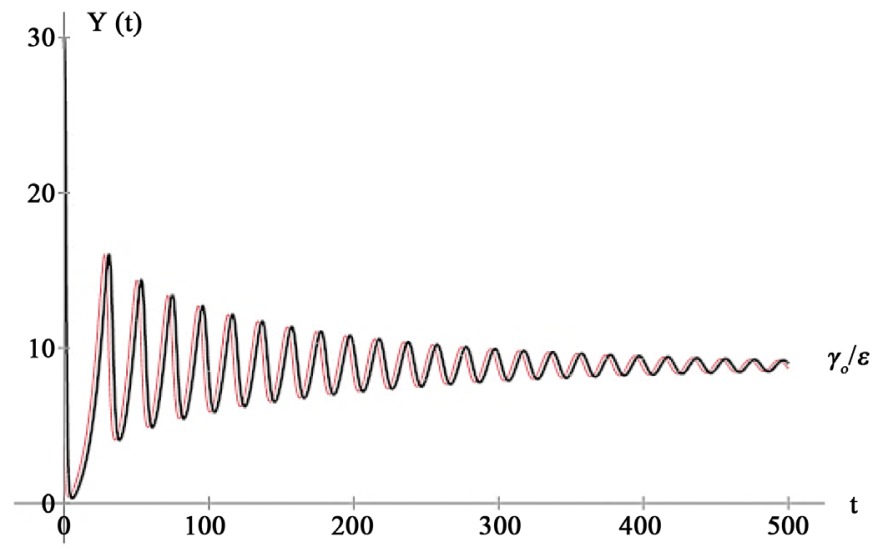

Figure 27. Effects of aging after stopping serial dilutions.

\subsection{Crosstalk and Beat Phenomena}

Official tests, performed in 1993, led to an unexpected fiasco (Section 5.2). It appeared, indeed, that the biological efficiency of EHDs was lost, while samples of pure water, needed for blind tests, turned out to be efficient. Benveniste was confronted to authorities, who concluded that the results did merely confirm their conviction that water memory is not a real and reproducible phenomenon. Since Benveniste knew that this was not true, because of numerous tests, he tried to understand the new observed facts. He realized that tubes with genuine EHDs of active molecules had been placed during some time near tubes that contained merely pure water. Benveniste thought therefore that the invisible structures, which are responsible for water memory, have to emit "signals", allowing them to transfer their biological efficiency to pure water. It was not clear why this information transfer was possible and why this could result in silencing authentic EHDs. However, Benveniste succeeded in proving, by means of purely empirical means, that the assumed signals do really exist.

He could detect them by merely putting a sample of some EHDs in a coil. This did yield an electric signal that could be amplified and applied to pure water. This involves physical processes that will be explained later on, but we know already that biologically active molecules stimulate their specific receptors by 
means of an oscillating electric field and resonance effects. Trimmed chains of WPs allow for standing waves and create an oscillating electric field that has the same effect inside EHDs. Normal interactions between active molecules and their receptors can also be bypassed (Figure 20).

We noted that resonance effects allow for some tolerance. It is thus sufficient that $f \approx f_{1} \approx f_{r}$, where $f_{1}$ is determined by the length $L$ of trimmed chains $\left(f_{1}=\right.$ $v / 2 L)$. However, it follows from Lo's measurements that $L=N D$, where $N \approx 20$. The fundamental frequency $f_{1}$ for standing waves will thus not always be precisely equal to the frequency $f$ that is characteristic of the chosen type of active molecules. It can happen, for instance, that the chosen type of active molecules did initially create attached trimmed chains where $N=20$ or $N=21$. Autogeneration of equally trimmed chains of WPs during successive dilutions is governed by a collective electric field that oscillates then at one of these frequencies. EHDs are thus able to "breed" identically trimmed chains. However, the resulting "strains" can be slightly different for two EHDs of the same substance. This is irrelevant when $f \approx f_{1} \approx f_{r}$. However, two EHDs with trimmed chains of nearly equal length produce electric fields of slightly different frequencies $\left(f_{ \pm}=f_{o} \pm \Delta f\right.$, where $\Delta f \ll f_{o}$ ). When these EHDs are contained in vessels that are put side by side, these fields are superposed. The resulting electric field does then oscillate at the average frequency $f_{o}$ with a modulated amplitude. Indeed,

$$
\begin{aligned}
& \cos \left(f_{o}-\Delta f\right) t+\cos \left(f_{o}+\Delta f\right) t=2 \cos \left(f_{o} t\right) \cos (\Delta f) t \\
& \cos \left(f_{o}-\Delta f\right) t-\cos \left(f_{o}+\Delta f\right) t=2 \sin \left(f_{o} t\right) \sin (\Delta f) t
\end{aligned}
$$

This beat phenomenon is well-known in acoustics. Even when the proximity of two slightly different EHDs was only temporary, their already strong electric fields generate together both types of trimmed chains. When this happened only during a short time, they will continue to produce both types of chains and therefore "mixed signals". Their biological efficiency will vary in a periodic way, but at a very slow pace. The relative phase of the superposed fields is also important, since the sum of two signals of equal amplitude will double their amplitude of oscillation. For the difference, the signals annihilate one another, but become strong again from time to time.

Momentary proximity of a genuine EHD with pure water can create there a small number of trimmed chains that resonate at one of the two possible frequencies. The sample of pure water becomes biologically active by "breeding" always the same strain of trimmed chains of Water pearls. Although Benveniste ignored the underlying mechanism, he had discovered that information transfer is possible. It proved the existence of signals and that they were responsible for water memory. Nevertheless, these signals had also extremely disconcerting effects. Beauvais used the term of "coherent discordances" to designate "wild transfers" and the fact that one operator did even "erase" the imprint [10] (p. 429, 450 and 453). These facts were enigmatic, but reproducible. For us, these parasitic effects do corroborate the theory of WPs. 


\subsection{Confirmations by Physico-Chemical Measurements}

Vittorio Elia and his collaborators performed remarkable experiments with the "long term goal" of clarifying the problem of water memory [46]. They began in 1999 with measuring the heat of mixing when EHDs of active molecules (like $\mathrm{As}_{4} \mathrm{~S}_{4}$ ) were dissolved in twice distillated water. It appeared that addition of EHDs of $\mathrm{NaOH}$ resulted in energy release. It was concluded that EHDs contain aggregates of water molecules that are able to create new ones. Elia insisted on the fact that these structures have the capacity of "self-organization". In terms of WPs, we can say that the added ions do also create trimmed chains of WPs. They are different, but bonds do always correspond to negative energy states. Mixing had thus to liberate more energy in the form of measurable heat.

The same team measured also the electric conductivity $\chi$ at $25^{\circ} \mathrm{C}$. Dissociation of $\mathrm{NaOH}$ produced ions that contributed to the measured electric conductivity. Ions liberated from the walls of the vessels did that also, but addition of EHDs of active molecules produced a significant excess conductivity $\chi^{\mathrm{E}}$. Since it was always proportional to the measured heat of mixing, both phenomena had a common cause [47]. The added active molecules increased the concentration of trimmed chains and thus also the electric conductivity inside molecular chains by the von Grotthuss mechanism.

Elia and his team made two other remarkable discoveries by measuring the excess conductivity $\chi^{E}$ for homeopathic dilutions of Arnica Montana during aging. The active substance is helenalin, $\left(\mathrm{C}_{15} \mathrm{H}_{18} \mathrm{O}_{4}\right.$, containing charged oxygen atoms). Its EHDs displayed wave-like variations of $\mathcal{X}^{\mathrm{E}}$, but at an extremely slow pace [48]. In 4.4 years, there appeared only 4 peaks, which had nearly the same form, but their amplitude increased [49] [50]. We can now understand this fact in terms of beat phenomena. It appeared also that the excess conductivity $\chi^{\mathrm{E}}$ was much greater for smaller volumes of EHDs. Initial conditions are usually irrelevant for aging (Figure 27), but $Y(t) \rightarrow \gamma_{o} / \mathcal{\varepsilon}$. This value will thus be increased when the probability $\varepsilon$ for creating associated chains is reduced by surface effects.

\section{More Evidence of Ferroelectric Water Pearls}

\subsection{The Measured Frequency Spectrum}

Benveniste discovered that EHDs of biologically active molecules produce "signals" that can be detected by means of a coil. The output was an electric tension that could easily be amplified and stored in analogical or digital form. The waveform was similar to that of noise, resulting from a superposition of simpler signals, randomly shifted with respect to one another. The predicted spectrum is a harmonic one:

$$
f_{s}=s f_{1} \text { where } s=1,2,3, \cdots \ll N
$$

This results from (9), which is an approximation of (10). Montagnier did publically show the results of Fourier analysis, nicely displayed on a computer screen [13]. The frequency spectrum did correspond to equidistant bell-shaped peaks. 
Moreover, their heights varied in the course of successive dilutions. Montagnier's patent US2010323391 contains the spectrum obtained by Fast Fourier Analysis [51]. It yields clearly separated spectral lines for shorter extracts. For a particular type of active molecules, the reported values were

$$
f_{s}=1000,2000,3000,4100,5100 \text { and } 5500 \mathrm{~Hz}
$$

The three lowest values do precisely correspond to (16). Since higher frequencies than $f_{1}$ result from molecular agitation in liquid water, they are excited with decreasing intensities. This implies greeter uncertainties. Important results of normal Fourier analysis were presented in an article [14], even for several substances and different stages of dilutions. They are especially interesting, like hieroglyphs, when we understand their meaning. For the first dilutions, there was only one great peak at the fundamental frequency $f_{1}$. We can attribute it to trimmed chains that are still attached to active molecules. Further dilutions led to the appearance of additional peaks, according to (16). Their average height increased during successive dilutions, but was maximal for dilutions D-9 to D-12. It did strongly decrease for D-13.

These facts agree with Figure 24 and confirm the predicted dissymmetry of peaks of activity. For the purpose of these authors, it was not necessary to continue the sequence of dilutions. They wanted only to get EHDs of maximal efficiency. It could be reliably predicted by observing the height of the peaks at higher frequencies. It corresponds, indeed, to the first stage of successive dilutions where $Y(t)$ and the biological efficiency are maximal. It may be astonishing that the peaks for some harmonic frequencies $f_{s}$ were depressed, but this due to interference effects, also for musical instruments [52].

\subsection{Water "Balls" and Their Alignments}

S.Y. Lo measured the diameter $(D=15 \mathrm{~nm})$ of WPs. The second group of structures had variable sizes of about $300 \mathrm{~nm}$. This yields the length $L$ of chains of WPs. Montagnier and his collaborators tried to measure the size of the required information carriers of water memory by means of filters. This method suggested a size between 20 and $100 \mathrm{~nm}$ [15]. No one knew that the carriers of water memory are single chains of WPs and can thus be broken by filtering. If $L=N D$ were always shorter than $100 \mathrm{~nm}$, these chains would contain at most $N=7$ WPs. The resolution for distinguishing different types of active molecules would then be too small to be efficient. By the way, the measured values of $L=\lambda / 2 \approx$ $300 \mathrm{~nm}$ and $f_{1}=\mathrm{v} / \lambda \approx 1 \mathrm{kHz}$, allow us to determine the velocity $v=2 L / f_{1} \approx 0.6$ $\mathrm{nm} / \mathrm{s}$ for any chain of WPs.

Lo found also by means of interference measurements a third group of supramolecular structures. Their size was much more variable, but of the order of $3000 \mathrm{~nm}$ [40]. The X-ray spectrum confirmed that they were only constituted of water molecules, even without silicon contaminations from the vessels. These structures could be created by solutions of different types of initiators, but required high concentrations of chains of WPs. Excitation by UV light produced 
fluorescence at $298 \mathrm{~nm}$, which is absent for pure water and ice. These "water balls" survived after short heating at $80^{\circ} \mathrm{C}$, but were decomposed by vigorous agitation. They were then spontaneously reassembled after about 15 min. Figure 28 reproduces, with kind permission of World Scientific Publishing Company, two pictures that were published the same year [53].

The left image proves that these balls tend to be aligned and the right image that they can be deformed. We propose therefore that water balls are constituted of chains of WPs, loosely bound to one another with global quasi-ferroelectric ordering. Water balls are thus dipolar, but contain also water molecules that can be expelled. They were said to be examples of "soft matter". Lo discovered also that when these balls are very numerous, they constitute extremely long alignments, visible by optical microscopy [54]. Spectacular images of these alignments were published in 2009 and reproduced in a book [55]. It gathers all articles of Lo and his collaborators concerning the discovery and empirical analysis of structured water. These images are also contained in the open-access article of Mae-Wan Ho [56].

It is very remarkable that these alignments are branching-off sideways, always at the same angle of $78^{\circ}$. Figure 29 shows that this astonishing fact can be explained. Since water balls contain chains of WPs, they have to be attached to one another at points, where all effective dipoles are oriented toward the center of neighboring water molecules. These dipoles are represented by red arrows, but parallel molecular chains are shifted with respect to one another by the distance $a$.
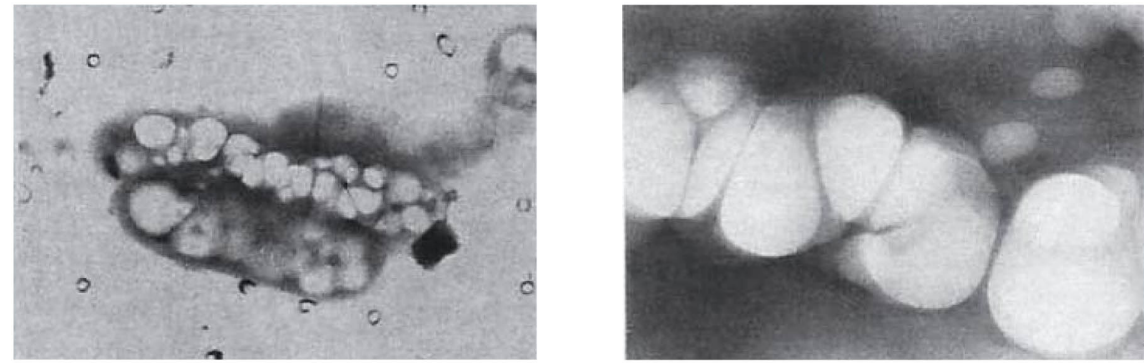

Figure 28. Transmission electron-microscopies, realized by Lo [51]. They show deformable "balls", constituted of chains of water pearls.

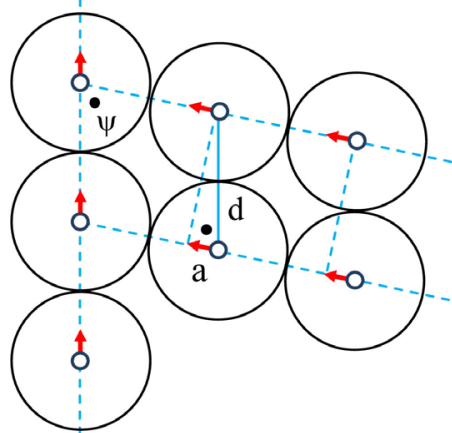

Figure 29. Chains of water molecules allow for strong bonds when they meet one another at the angle $\psi=78^{\circ}$. 
Since the centers of adjacent water molecules are separated by the distance $d$, molecular chains meet one another at an angle $\psi$, which appears in Figure 29 at two different places, indicated by dots. It follows that $\cos \psi=a / d=0.6 / 2.9$. Thus, $\psi=78^{\circ}$. This result does strongly confirm the validity of the proposed theory.

\subsection{Proposed Explanation of Water Bridges}

What would happen if liquid water were subjected to very strong electric fields? This question has been raised long ago by the British lawyer Willian George Armstrong, who became a respected engineer, inventor and scientist. He had a powerful source of high electrostatic potential differences and used it to find out if they produce very intense electric currents in liquid water. He knew that these currents would result from motions of $\mathrm{H}^{+}$and $\mathrm{OH}^{-}$ions, but he discovered surprising facts. He presented them in 1893 to a general audience as being entertaining, but mentioned that they might "be interesting to experts" [57]. Actually, he filled two wine glasses up to the rim with pure water and put them in contact with one another. He immersed a long cotton thread in one of them and introduced one wetted end of it in the other glass. He was amazed to see that a high potential difference between water in the adjacent glasses did transfer the whole cotton thread to the other glass. Why did this happen?

The spongy cotton thread had to be entrained by motions of charges. Since $\mathrm{OH}^{-}$ions have a greater mass than $\mathrm{H}^{+}$ions, the thread should move towards the positive pole. This is what Armstrong observed, but the water level remained constant in both glasses. This was confirmed when Armstrong used a vessel where a thin tube allowed to see more precisely any variation of this level. Hementioned that during a few seconds after the complete transfer of the cotton thread, a "rope of water" remained suspended between the two glasses. When Elmar Fuchs was studying physics in Austria, he heard about this phenomenon and reproduced it with a source that could sustain high currents at $15 \mathrm{kV}$, for instance.

The first results were published in 2007. He found that the cotton thread was not necessary to produce a "floating bridge" of liquid water [58]. It remained stable as long as the high electric field was applied. This phenomenon did raise a fundamental problem [59]: how is it possible that a strong electric field modifies the interaction between water molecules in the liquid state so much that it becomes able to resist gravity? The generation of the water bridge was itself a complex process. At first, the water surface became agitated in both vessels. Then, a very thin filament of liquid water established contact, but it lasted only a fraction of a second. Its diameter was then suddenly increased to yield a cylinder of circular section, indicating strong surface tension. It remained suspended in air like a catenary, even when its length was slowly increased up to $2.5 \mathrm{~cm}$ at $25 \mathrm{kV}$. Fuchs started then a systematic study of this strange phenomenon with various collaborators, to discover the cause of this "new state" of liquid water.

Since water bridges provided direct visual evidence, it would have been diffi- 
cult to negate the reality of these observations, but the basic problem was the same as for water memory. What could be observed? In regard to electric conductivity, it was confirmed that the transport of charges is bidirectional [60]. Protons $\left(\mathrm{H}^{+}\right)$and proton-holes $\left(\mathrm{OH}^{-}\right)$are passing through the bridge. By measuring the complex impedance between $100 \mathrm{~Hz}$ and $10 \mathrm{MHz}$, it appeared even that the charges were moving like nearly-free conduction electrons in metals [61]. Raman scattering indicated also that vibrational modes in liquid water were not modified with respect to usual ones in bulk water [62]. Charge transport was due to the von Grotthuss mechanism, but we have to solve two problems. 1) Why is cohesion of water molecules in the liquid state enormously increased by applying a very intense electric field? 2) How can many $\mathrm{H}^{+}$and $\mathrm{OH}^{-}$ions move in opposite direction, without hindering one another?

The first question has to be related to the higher density of liquid water near the surface of the bridge. This was proven by X-ray scattering for two-dimensional beams of submillimeter extension [63]. Since neutron scattering is sensible to hydrogen atoms, it yields complementary results [64]. The stability of water bridges has been attributed to higher surface tension [65], but that is not sufficient, since longitudinal stretching of the "liquid cable" requires greater bond-strength along this direction. We propose that the stability of water bridges is due to the formation of very long chains of WPS.

We can then understand the sequence of events. At first the water surface became agitated in both beakers, since growing chains were formed near the electrodes and then moving around, until contact was established between water in both vessels. At first, there were only few chains, constituting a capillary bridge, but once the way was open, more and more chains of WPs were rapidly formed by the very intense electric field. Statistical fluctuations of the traffic of protons and proton-holes along the thin bundle of chains of WPs led to repulsion. It became more efficient to push parallel chains towards the surface of the liquid bridge. The capillary bridge was, indeed, replaced by a thicker one to achieve a new equilibrium. Since WPs are constituted of more densely packed water molecules than in liquid water, chains of WPs, situated at the surface of water bridges should there produce a greater density. This explains also the observed birefringence for linearly polarized light. Moreover, higher potential differences and greater electric currents require more chains of WPs at the surface of the liquid bridge. This should increase the diameter of the bridge and does agree with observations [66].

An increase of the applied potential difference allows also for a greater length of the catenary. This fact is due to the polarization of water molecules, as shown in Figure 30. The left part represents a molecular chain like that of Figure 10 for a moderate electric field, while the right part indicates that a very intense electric field $\mathrm{E}$ does strongly polarize the water molecules. The usual angle of $106^{\circ}$ between $\mathrm{OH}$ bonds is reduced and the distance between proton pairs and the negative cores of oxygen atoms is also reduced by deformations of the usual, nearly 

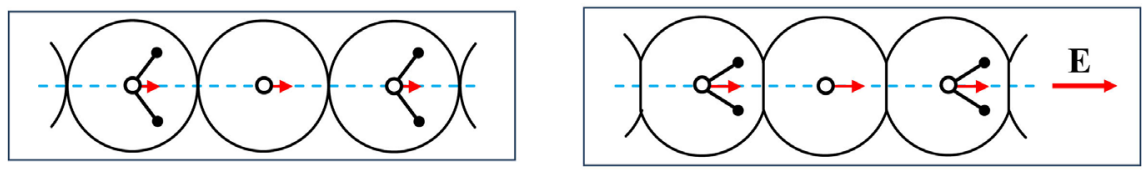

Figure 30. Aligned water molecules get more tightly bound by a strong electric field E.

spherical shape. Very strong polarization of water molecules would thus increase the mutual attraction of neighboring water molecules. This is also true when the bonds are due to intramolecular exchange effects (Figure 6). Electric conduction by the von Grotthuss mechanism would thus be facilitated, but regulation of opposite traffic of protons and proton holes is imperative for very intense electric currents passing though the water bridge.

Some observations indicated that this might happen in a helicoidally coordinated way [65]. This makes sense, when we adopt the model of Figure 31. Groups of positive and negative charges are there represented by red and blue dots. They are distributed in a plane, to constitute a lattice like that of ionic crystals, but this plane rotates around the axis, while the charges are advancing along different lanes, represented by red and blue lines. [This double helix is similar to Da Vinci's famous stair case of the chateau of Chambord in France. It contains two flights, allowing for simultaneous upward and downward traffic.]

\subsection{Lack of Evidence for Coherence Domains}

Emilio Del Giudice et al. proposed in 1988 a bold hypothesis concerning properties of bulk liquid water at very small scales [67]. Basically, they assumed that inside some water molecules that belong to small spherical domains, electrons can remain in an exited state. Since the ionization energy of water molecules is 12.6 $\mathrm{eV}$, they considered excitation to an energy level at $12.06 \mathrm{eV}$. To keep the electrons in this weakly bound state, they assumed constant emission and reabsorption of virtual photons, trapped inside the small spherical volume. This required that electrons should be accumulated at the surface of the sphere, while positive charges had to be expelled and would be homogeneously distributed in the surrounding liquid water. The accumulated electrons should constitute a barrier that reflects virtual photons. It was also necessary to postulate that the ensemble of excited electrons inside such a sphere are oscillating in phase with the EM field of all virtual photons. Because of the selected excitation energy, the wavelength would be close to $100 \mathrm{~nm}$ and determine the size of these coherence domains (CDs). It was estimated that they contain about 1000 water molecules, since only a fraction of the internal electrons would be excited.

Although this theory was initially conceived for bulk water [68], it was assumed later-on that it should even account for exclusion zones, water bridges and water memory. Because of the prestige of quantum field theory and the need of a rational justification of the mysterious phenomenon of water memory, the theory of CDs had increasing impact. However, we have also to mention what happened in 1993. It had already been decided that Benveniste's Unit 200 would 


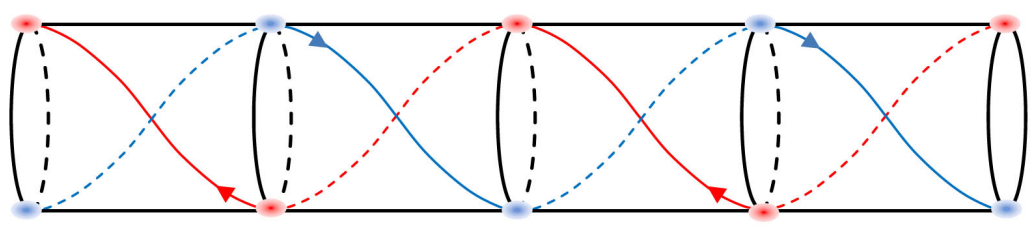

Figure 31. Opposite motions of bunched charges along a double helix.

be closed, but he found a more objective method for detecting the biological efficiency of EHDs. He was thus allowed to prove the reality of water memory by means of blind experimentation, subjected to rigorous control of Georges Charpak. He was the 1992 Nobel Prize laureate in physics, since he invented a new type of particle detectors for CERN. Benveniste tried to convince him, by referring to the article of Del Giudice et al. [67].

Since this article concerned condensed matter physics, Charpak asked the opinion of Pierre-Gilles de Gennes, also Nobel Prize winner in physics. He answered that this theory is "worth nothing", since it is based on "false hypotheses" ([6], pp. 154, 168). Beauvais mentions that de Gennes had asked Philip Nozières ([13], pp. 395, 398 and 402). He was the top theoretical physicist in France for new properties of condensed matter. Since such a harsh judgment might have been influenced by controversies, we have to know why the concept of CDs has been proposed. The theoretical physicist Giuliano Preparata (1942-2000) made important contributions to elementary particle physics, before becoming interested in applying quantum electrodynamics (QED) to condensed matter physics [68]. He viewed the existence of CDs as being a general property of pure liquid water in the sense of a "two fluid picture". He stated even that "the concept of H-bonds is merely phenomenological", since liquid water should rather be viewed as a "condensed vapor" than "molten ice" ([68], pp. 38, 196, 197). Liquid water would locally be condensed, before becoming ice.

Preparata was coauthor of the official article on CDs [67], presenting the same idea in Physical Review Letters. The primary author, Emilio Del Giudice (1940-2014), was one of the pioneers of string theories and explained why he got interested in CDs [69]. Since his youth, he did feel that the whole universe constitutes a profoundly integrated entity and studying physics, he developed a sense of wonder in regard to quantum mechanics (QM). He got especially interested in "entanglement". Since this concept provided his motivation for considering CDs, we have to clarify its origin and physical meaning.

Albert Einstein had contributed to the development of quantum mechanics, but in 1927 he learned about new ideas and discussed them with Niels Bohr at the Solvay conference in Brussels. He perceived very keenly that this theory attributed peculiar properties to measurements. He developed this idea in the famous EPR article of 1935, by means of a thought experiment. We describe it in equivalent terms, by considering two particles that have a property that can be precisely measured, but allows only for two possible values: +1 and -1 . Since QM accounts for limited knowledge, we can define a state where it is only 
known that the values \pm 1 are equally probable. It is then sufficient to determine by means of a new measurement that the value is +1 for one particle, to be instantly sure that it is -1 for the other particle. It does not matter how far these particles are separated from one another at that instant.

In classical physics, that would require the existence of a physical link. Einstein asked therefore: are such "spooky actions" at a distance physically possible or not? He did only raise the problem, while Schrödinger had developed a theory where causal relations were preserved for the propagation of wave functions in space and time. He insisted on the need of causal relations and did not like the idea of "quantum jumps". They do not allow for further analysis. Since Einstein had described in a vivid way that some measurements seem to imply universal connections, he coined the word of "entanglement" to account for them. They would require that instantaneous information transfer is possible at any distance, although this is excluded by the theory of relativity. Actually, it is sufficient to accept that QM is a theory of possible knowledge, limited by universal restrictions that Nature imposes on some measurements.

Classical physics postulated that totally precise, simultaneous knowledge of positions and velocities is possible. It implied continuity and strict causality, but this assumption has to be corrected. The basic paradigm of QM is that the constant $h$ imposes irreducible uncertainties. The concept of "virtual photons" results from the fact that during short time intervals $\Delta t$, the energy of a system can only be known with a minimal uncertainty $(\Delta E \approx h / \Delta t)$. Since the theory of relativity imposes that the energy $\left(m c^{2}\right)$ of particles of given mass is finite, the number of identical particles cannot be precisely known during short time intervals. This applies also to photons and leads to some observable effects.

Del Giudice was convinced that virtual photons are even able to establish a link between physical systems when they are separated by arbitrarily great distances. He expressed this idea [70] by stating that telepathic communications are possible, but he knew that physical theories are only justified by their agreement with observations. He tried thus to show that the concept of CDs accounts for unexplained phenomena, like water memory, exclusion zones and the stability of floating water bridges. This idea was diligently propagated, but does it really account for water memory?

Bellavite and other health specialists at Italian universities reviewed in 2013 the status of research concerning EHDs [71]. They concluded that "evidence strongly supports the notion that the structuring of water and its solutes plays a fundamental role", but the mechanism is not yet understood. Two hypotheses had been proposed: hydrogen bonded clathrates and coherence domains. After examining the evidence, they concluded that "none is fully convincing". The concept of ferroelectric water pearls was not yet known. It explains essential properties of EHDs, like the observed quasi-periodic variations of their biological efficiency, the measured frequency spectrum of signals created by local structures and even the angle of $78^{\circ}$ between alignments of water balls. In his regard, we have to mention that Mae-Wan Ho tried to explain alignments of 
water balls [56], by assuming that expelled protons are not homogeneously distributed in liquid water. They could be more concentrated between the charged CDs, but that is not sufficient to explain the observed angle of $78^{\circ}$ for chains of water balls.

Benveniste was sure that water memory is real, because of the often verified quasi-periodicity of the biological efficiency of EHDs and detection of signals. It was thus obvious that a physical explanation had to be possible. Since CDs seemed to offer an explanation, Montagnier accepted the help of scientists who advocated this approach. They stated even that water memory involves the "gauge theory paradigm of quantum fields" and "the framework of spontaneously broken gauge symmetry theories" [72]. These words are familiar to those who know the jargon of quantum electrodynamics (QED). They are impressive, but are they necessary? We recall that Thomas Kuhn distinguished three periods in the process of scientific revolutions [45]. At first, the observed anomaly is declared to be impossible, since it does not agree with conventional knowledge. When it is confirmed and cannot simply be denied anymore, it comes to a "crisis", where any hypothetical explanation may be proposed.

The theoretical chemist Tamar Yinnon published a series of articles, where the concept of CDs was elaborated, by postulating the existence of various types of these structures. He presented them in 2015 as being "predictions of QED", but we found only a catalogue of assumed structures [73]. The original CDs were renamed $C D_{\text {elec }}$. Their diameter would thus be about $100 \mathrm{~nm}$. Greater structures, called $\mathrm{CD}_{\text {plasma }}$, were assumed to contain some molecules of the solvated substance, but also more water molecules. Their effective dipoles would be oriented by the electrically charged active molecules or ions inside these domains. The resulting hydration spheres were said to be subjected to (monopolar) plasma oscillations with overall coherence. The size of these domains would be of the order of $1000 \mathrm{~nm}=1 \mu \mathrm{m}$. A third type of CDs should reach sizes of $10-100 \mu \mathrm{m}$. They were called $\mathrm{CD}_{\text {rot }}$ and assumed to contain only water molecules, but all of them would have nearly parallel effective dipoles. They would be large elongated ferroelectric particles. Moreover, agglomerated $\mathrm{CD}_{\text {plasma }}$ and $\mathrm{CD}_{\text {rot }}$ entities constitute "supra-domains".

\subsection{Forced Oscillations of Water Molecules in EHDs}

Instead of commenting these respectable attempts to find an explanation of water memory, we continue to collect experimental results and to test the validity of the concept of WPs. Adriana de Miranda measured, for instance, the dielectric response of water molecules that interact with supramolecular structures [74]. She used 14 lots of $\mathrm{LiCl}$, prepared by pharmaceutical laboratories and determined the spectral distribution of the real and imaginary parts of the impedance in the frequency range of $1 \mathrm{kHz}-13 \mathrm{MHz}$. She defined these variables by means of an equivalent circuit and compared the results to those of the measured dielectric response of EHDs of $\mathrm{H}_{2} \mathrm{O}$ molecules in pure water, prepared in the same way. To understand the underlying physical processes, we consider the 
response of the effective dipoles of water molecules to an oscillating electric field at various frequencies. The center of these molecules remains practically motionless, while the positive tip of the effective dipole is displaced by a small distance $u(t)$ along the direction of the applied electric field. When $n$ is the density of water molecules, the instantaneous polarization density is $P(t)=n 2 q u(t)$, where the displacement $u$ is subjected to the equation of motion

$$
\dot{u}=-u / \tau+(q / m) E(t)
$$

Indeed, $2 q$ is the charge and $2 m$ the effective mass of the pair of protons. When the applied electric field $E(t)$ is suddenly extinguished at the instant $t=0$, $u(t)=u(0) \exp (-t / \tau)$. The value of the relaxation time $\tau$ depends on all possible interactions in bulk water. For an electric field that oscillates with some constant amplitude at a given (angular) frequency $\omega$, we get $E(t)=2 E_{\omega} \cos (\omega t)=E_{\omega} \exp (-i \omega t)+c . c$ and the instantaneous polarization density is $P(t)=\beta(\omega) E_{\omega} \exp (-i \omega t)+c . c$. The complex conjugate (c.c) requires merely that $\omega$ is replaced by $-\omega$. It follows that

$$
\beta(\omega)=\frac{n(2 q)^{2} / 2 m}{\gamma-i \omega}=\frac{\beta(0)}{1-i \omega \tau}=\frac{\beta(0)(1+i \omega \tau)}{1+(\omega \tau)^{2}}=\beta_{1}(\omega)+i \beta_{2}(\omega)
$$

The function $\beta_{1}(\omega)$ specifies the average orientation of effective dipoles. At low frequencies $(\omega \tau \ll 1)$, they are oriented along the direction of the applied electric field. The polarization drops quite suddenly when $\omega \tau \approx 1$ and is reduced to zero when $\omega \tau \gg 1$. The imposed rhythm is too fast to allow the molecules to follow, because of friction. The imaginary part $\beta_{2}(\omega)$ varies like $x /\left(1+x^{2}\right)$, when $x=\omega \tau$. This yields a peak that is centered on $x=1$. The function $\beta_{2}(\omega)$ describes the energy-loss of water molecules, because of friction. It is maximal when $\omega=$ $1 / \tau$. Since increased friction leads to lower values $\tau$, a higher frequency is then required to achieve great energy losses. In $\mathrm{QM}$, higher frequencies correspond to higher energies and in QED even quasi-static electric forces are due to exchanges of virtual photons. Miranda found that the values of relaxation times were situated between 40 and $100 \mathrm{kHz}$ for EHDs of $\mathrm{LiCl}$ and $\mathrm{H}_{2} \mathrm{O}$ molecules in pure water. This means for us that friction resulted from the creation of trimmed chains of WPs that had different lengths. However, the height of the peak for the energy-loss function varied in the course of successive dilutions of $\mathrm{LiCl}$ in such a way that it was maximal for D-9. Since friction is proportional to the concentration $Y(t)$ of single trimmed chains of WPs, this agrees with our theoretical predictions (Figure 24).

\subsection{Discovery of Highly Remarkable Resonances}

Since we expected that stationary waves on trimmed chains of WPs can be excited by an electric field, we did search relevant data and found the results of the Indian electrical engineer Chitta Ranjan Mahata [75] [76]. He had witnessed the healing power of homeopathy and wanted to understand how this is possible. Studying the literature [77], he discovered the proposition of Barnard [78]. $\mathrm{He}$ 
declared already in 1965 that homeopathy is in need of a "surer foundation" than to refer to positive results. This should be possible, since homeopathy requires that EHDs create substitutes of the initially dissolved active molecules. Since they should merely be constituted of water molecules, Barnard thought that they might "join up to form long molecular chains". This idea was probably suggested by the concept of polywater, which was in vogue at that time. Barnard proposed that these molecular chains may be wrapped around active molecules and that their length would thus provide the required specificity.

We were surprised that some important ideas had already been formulated more than 20 years before Benveniste's discovery. Specificity was even related to the length of local structures, composed of water molecules. Barnard was aware of the hypothetical nature of this proposition, but insisted that it indicates "the kind of experimental research in physics and chemistry needed now to establish the truth of homeopathy." Mahata realized that he could test this hypothesis, since ordered molecular groups in liquid water should lead to resonance effects.

Before describing and explaining his results, we have to mention that Barnard and Stevenson provided more details in another article [79]. They reviewed what was known about properties of EHDs, prepared for therapeutic purposes. It had already been established that their capacity of healing is subjected to "rhythmic variations" in the course of successive dilutions. It was also mentioned that biologically active molecules contain electric charges and that Alphonse Gay had developed in the early $1950^{\text {th }}$ a method for measuring the dielectric response of EHDs [80]. It was complicated, but sufficient to identify EHDs of $\mathrm{NaCl}$ after 27 centesimal dilutions. [We thank Dr. van Wassenhoven for his help to get access to this paper.]

Mahata was not aware of these measurements and developed with his collaborators a much more efficient technique. In 2007, it was ready [81] and in 2010, they presented results for the spectral distribution of the real and imaginary parts of the dielectric constant [75] in a large frequency domain $(100 \mathrm{kHz}-50$ $\mathrm{MHz}$ ). They found typical features of resonances for preparations of Cuprum met, but also for pure water. In 2013, they provided more results [76] and did interpret them in terms of Barnard's model. There were resonances, but we noted four perplexing anomalies:

1) These resonance frequencies were always very high: about $25 \mathrm{MHz}$ instead of the expected ones at about $1 \mathrm{kHz}$.

2) There was only one resonance frequency, without harmonics.

3) A resonance was even observed for pure water.

4) The energy-loss functions were not symmetric, as required for usual resonances.

This did not correspond to expectations for single chains of WPs, but we realized that the third anomaly could be explained, since pure water contains $\mathrm{H}^{+}$ and $\mathrm{OH}^{-}$ions. Their electric fields might be sufficient to produce at least isolated water pearls. The observed high value of the resonance frequency would then 
require a very strong restoring force. For single WPs, it could only be due to their surface charges. They are represented by red and blue rims in Figure 31 . For low resonance frequencies $(\sim 1 \mathrm{kHz})$, it was licit to assume that the oriented water molecules remain oriented towards the surface of the WP. However, at high frequencies $(\sim 25 \mathrm{MHz})$, the solid substrate is moving too fast to allow them to be reoriented so rapidly. Miranda's measurements indicated, indeed, that reorientation of water molecules occurs at lower frequencies. Because of the proximity and spatial distribution of the internal and external charges, this would then lead to a very strong restoring force.

We test the validity of this hypothesis, by comparing its logical consequences to Mahata's experimental results. The component $\mathrm{E}(\mathrm{t})$ of the applied electric field, which is normal to the symmetry axis of the WP sets the positive and negative poles of the WP in forced oscillation. The equation of motion for small displacements $\mathrm{u}_{\mathrm{o}}(\mathrm{t})$ of these poles is

$$
\ddot{u}_{o}+\Omega^{2} u_{o}+\gamma \dot{u}_{o}=C_{\omega} \exp (-i \omega t)
$$

The (angular) resonance frequency $\Omega$ is determined by the strong restoring force and the effective inertial mass of the poles. Energy losses by friction are characterized by $\gamma$, while $C_{\omega}$ is proportional to the amplitude of the electric field $\mathrm{E}(\mathrm{t})$. Thus, $u_{o}(t)=B(\omega) E_{\omega} \exp (-i \omega t)$ for constantly forced oscillations. The polarizability $\beta(\omega)$ of pure water that contains a given concentration of isolated WPs is proportional to $B(\omega)$. When we normalize $\beta(\omega)$ to get always the same static polarizability $\beta(0)=1$, it follows that

$$
\beta(\omega)=\frac{\Omega^{2}}{\Omega^{2}-\omega^{2}-i \omega \gamma} \approx \frac{\Omega^{2}}{\Omega^{2}-\omega^{2}}+\frac{i \Omega \Gamma / 2}{\Omega^{2}-\omega^{2}-\Gamma^{2}}
$$

We simplified the real part $\beta_{1}(\omega)$ to insist on the fact that without friction, this function would diverge when $\omega=\Omega$. At low frequencie $(\omega \leq \Omega)$, the average orientation of an ensemble of isolated WPs is identical to that of the applied electric field. It is opposite when $\omega \geq \Omega$, but WPs cannot follow the applied field when $\omega \gg \Omega$. The function $\beta_{2}(\omega)$ describes energy losses. They are maximal when $\omega \approx \Omega$. The peak is symmetric and we can set $\gamma / 2=\Gamma$. The height of the peak is then $\Omega / 2 \Gamma$ and its width at half height is equal to $\Gamma$. Figure 32 shows (in black) the frequency dependence of $\beta_{1}$ and (in red) the spectral distribution of the energy-loss function $\beta_{2}$ when the resonance frequency $\mathrm{f}=25 \mathrm{MHz}$ and $\Gamma=5$ $\mathrm{MHz}$.

These results had to be expected for a normal resonance, but Mahata's experimental results for $\beta_{2}$ did correspond to curves like the blue one. The height of the observed peak has been adjusted in Figure 33 to coincide with the height of the normal peak and $\beta_{\text {2exp }} \rightarrow 0$ when $\omega \gg \Omega$. Measured energy-loss functions are thus asymmetric. This feature did also appear for EHDs of various biologically active substances. Only the values of $\Omega$ and $\Gamma$, as well as the mysterious "dip" of the energy loss functions were slightly different. All these facts will be explained later on (in Section 5.4). Before we do that, we continue the equally necessary search of more evidence. 


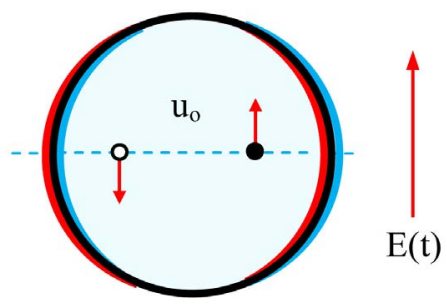

Figure 32. Rapid oscillatory rotations of a single water peal, excited by an electric field.

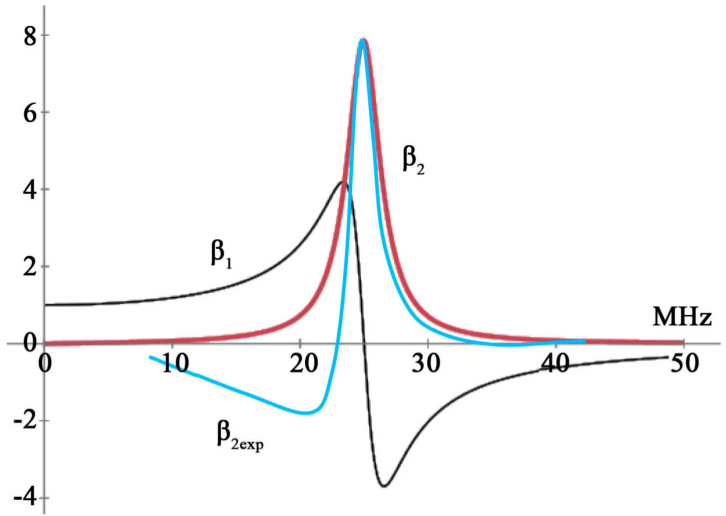

Figure 33. The spectral distributions of the real and the imaginary parts of the polarizability $\beta$, predicted for oscillators that resonate at $25 \mathrm{MHz}$. The blue curve represents typical results for Mahata's resonances.

\subsection{Complementary Experimental Evidence}

The Swiss biochemist Louis Rey did prove in 2003 that EHDs of $\mathrm{NaCl}$ and $\mathrm{LiCl}$ in ultrapure water produce local structures that are preserved after freezing [82]. He kept the EHDs during $24 \mathrm{~h}$ at $-20^{\circ} \mathrm{C}$ and reduced then the temperature to 77 $\mathrm{K}$. When he exposed the transparent disk to a high dose of ionizing radiation, most excited electrons did immediately return to their normal state in ice, but some of them were trapped in local defects. To leave enough time for diffusion of electrons, the ice remained during one week at $77 \mathrm{~K}$. After reaching equilibrium, the sample was warmed up at constant rate. The trapped electrons were thus progressively raised to states of higher energy, allowing for light emission. This thermoluminscence is a standard method for examining defects in insulators, since it determines the excitation energies that are required to liberate electrons from various traps.

There appeared a glow for $T \approx 120 \mathrm{~K}$ and a more intense one at $T \approx 166 \mathrm{~K}$, especially when $\mathrm{NaCl}$ had been dissolved in heavy water $\left(\mathrm{D}_{2} \mathrm{O}\right)$. Since electron traps were different for $\mathrm{NaCl}$ and $\mathrm{LiCl}$, at least some of them were due to $\mathrm{Na}^{+}$ and $\mathrm{Li}^{+}$ions. Rey concluded that these results prove "without any ambiguity" that liquid water has been structured in a lasting way. Since the glows appeared only when serial dilutions were followed by vigorous shaking, Rey thought that it might produce nanobubbles, attracted by ions [83]. The basic facts did not depend on this interpretation and were brilliantly confirmed by van Wijk et al. [84]. We can conclude that EHDs did produce trimmed chains of WPs, where 
the molecules are more closely packed and so strongly bound to one another that the ferroelectric crystallites do even subsist in normal ice (Figure 4).

Demangeat et al. studied the effects of EHDs by means of nuclear magnetic resonance [85]. Protons have indeed a magnetic moment that tends to be parallel or antiparallel to the direction of a constant magnetic field $\boldsymbol{B}_{o}$. The energy difference is proportional to the magnitude of this field and the transition can be stimulated by EM radiation of adequate frequency. When the excitation ceases, the system returns to its ground state, but there are two different relaxation times, $T_{1}$ and $T_{2}$, for components of the magnetic moment of protons along the direction the magnetic field $\boldsymbol{B}_{o}$ and perpendicular ones. The ratio $T_{1} / T_{2}$ depends on their environment. For EHDs, the results of measurements did prove that "water is a self-organizing system" [78]. Van Wassenhoven et al. presented more detailed results of NMR studies, confirming this conclusion [86] and providing additional information.

Other very important facts were discovered in Russia. They did prove that water molecules can also constitute stable structures without having to apply the standard procedure to get EHDs. Konovalov and Ryzhkina presented in 2014 a review [87], where they stated that the following conditions have to be satisfied: 1) only one type of molecules should be dissolved in pure liquid water at extremely low concentration, 2) these molecules have to carry an electric charge and 3) be subjected to low-frequency EM fields. This was verified for different types of molecules and the resulting structures were also observed by Atomic Force Microscopy (AFM). The authors insisted that these entities do not result from the formation of nanobubbles, but are associations of water molecules. This is essential to justify the concept of WPs, although the hypothesis of nanobubbles is closer to conventional ideas.

Burkin and his collaborators [88] performed light scattering measurements for very low concentrations of diluted molecules and interpreted their results in terms of bubble clusters around ions. This hypothesis leads to inconsistencies [89]. The Indian chemical engineer Chikramane and his collaborators used homeopathic drugs, prepared by means of EHDs of various metals ( $\mathrm{Au}, \mathrm{Ag}, \mathrm{Cu}, \mathrm{Sn}$, $\mathrm{Zn}$ and $\mathrm{Pt}$ ). They did yield local structures of sizes that ranged from 214 to 325 $\mathrm{nm}$. They could thus be trimmed chains of WPs of length $L \approx 300 \mathrm{~nm}$, but these authors claimed that $\mathrm{x}$-ray analysis demonstrated that they do contain particles of the initially dissolved metals. They assumed therefore that minute gas bubbles were formed during agitation and stabilized by attachment to small metal particles. Moreover, they thought that these entities rise to the surface and constitute there a thin layer that is reconstituted during subsequent dilutions. This would raise unanswered questions. NMR and other measurements of Van Wassenhoven for copper particles did not confirm this hypothesis [86]. Maybe, some procedures for preparing EHDs allow that charged metal particles get attached to WPs, because of their surface charges.

Demangeat thought also that vigorous agitation could produce nanobubbles. He proposed even that they might account for water memory by creating a "ste- 
reospecific shell" around active molecules [90]. How could these molds be detached, remain stable and allow for replication? Nevertheless, the concept of nanobubbles was attractive in the context of the "crisis", which resulted from the unsolved puzzle of water memory. It required a specific experimental investigation. The Slovenians Sedlák and Rak combined light scattering with rapid centrifugation and established that biologically active molecules at very low concentration produce real material entities [91]. Their measured sizes ranged from100 $\mathrm{nm}$ to $400 \mathrm{~nm}$ for various substances. It was explicitly noted that the mechanism of formation of these supramolecular structures and their nature were not yet known. However, the exclusion of nanobubbles and the reported sizes are compatible with trimmed chains of WPs.

Elia et al. produced supramolecular aggregates in pure water by repeated contact with a polymer, called Nafion [92]. It is strongly hydrophilic, because of its electrically charged sulfonate group $\left(-\mathrm{SO}_{3} \mathrm{H}\right)$. It produced structures in liquid water, subsisting after freeze-drying. In solution, they had a high electric conductivity, attributed to proton hopping. They exhibited UV absorption at 270 $\mathrm{nm}$ and modified IR absorption, associated with the $\mathrm{OH}$ stretching mode of vibration. Solid residues after evaporation displayed clustered particles of about 40 to $400 \mathrm{~nm}$. It appeared that these aggregates of water molecules produce circular dichroism [93]. This means that plane polarized light, decomposed in left and right circular polarized light, does not lead to equal absorption of these components. Dichroism indicates the presence of left-handed and right-handed structures, which can be tripoles in ferroelectric crystallites.

\section{New Types of Molecular Interactions}

\subsection{The Sense of Smell}

The most important consequence of water memory and its elucidation is that molecules do not only interact with one another by direct contact. This discovery belongs to a trend that began with trying to understand the sense of vision. John Dalton had described in 1794 his color blindness. Actually, he was unable to distinguish green from red. The British physician and physicist Thomas Young was intrigued by this anomaly, which led him to raise again the basic question: what is light? Newton had discovered that light of different colors can be separated by refraction. This could be explained by assuming that light is composed of particles, moving at constant velocity in any homogeneous, transparent medium. Why is it constant and depends on the medium remained mysterious, but refraction would then simply result from acceleration or deceleration at the interface.

However, Young discovered in 1801 that when light passes through two very narrow and close holes or slits, there appear dark fringes. He explained these results in terms of superposed "undulations", similar to those that can be observed on water surfaces. These interference effects led to the concept of light waves. Colors were then determined by their wavelength. They can be measured by 
means of gratings, but our eyes do not perform this kind of spectroscopy. Young realized that color vision requires only three types of receptors in our retina, mainly sensible to red, green and blue. Dalton had no green sensible receptors. These receptors had to absorb energy. [During about one century, it was assumed that light corresponds to waves. Actually, it is composed of photons, which are particles that carry energy and momentum, defined in terms of frequencies and wavelength. Photons behave even according to laws that are valid for waves. This synthesis transcends the idea of a dual nature of light.]

The English physiologist William Ogle tried to understand the sense of smell. Since anomalies could provide a clue, he collected and analyzed cases of "anosmia", i.e. partial or total loss of the sense of smell. He concluded in 1870 that odors are not perceived by means of chemical processes. It requires receptors that detect waves [94]. Ogle proposed that they should correspond to "undulations of the ether". [The concept of EM waves emerged only in 1873, through the development of Maxwell's theory. He gathered the laws of electricity and magnetisms, established for static or slowly varying electric and magnetic fields. $\mathrm{He}$ found that they are inconsistent when they are extrapolated to high frequencies, but harmony could be restored by adding a new physical law. It implied that electric and magnetic fields can constantly be transformed into one another, without needing the presence of electric charges. The theoretically predicted existence of electromagnetic waves was experimentally confirmed in 1887 by $\mathrm{Hei}$ nrich Hertz.]

Because of QM, it became also clear why molecules can emit photons of infrared light and that this type of spectroscopy allows us to distinguish different types of molecules from one another. Malcolm Dyson proposed therefore in 1938 that the sense of smell is due to receptors that detect vibrational frequencies of molecules by energy absorption [95]. How is that possible without complicated instruments? This problem has been solved in 1996 by the biophysicist Luca Turin. Being a specialist of perfumes, he wanted to understand how odorants can be perceived and differentiated. Since olfactory receptors of insects, animals and humans are very small, he was looking for very compact detectors. Thus, he explored the domain of solid state electronics. Eventually, he found that this can be achieved by means of inelastic electron tunneling [96]. He told the story of this discovery in a very interesting book [97].

The Japanese Leo Esaki had invented in 1957 an efficient diode. It was based on properties of two $\mathrm{n}$ or $\mathrm{p}$ type semiconductors, separated by a very thin gap. It blocks the passage of electrons or electron holes, when the conduction band on one side meets a forbidden band on the other side. However, these bands can be shifted with respect to one another by applying a potential difference. Charge carriers can then pass through the intermediate potential barrier by wave-mechanical tunneling. The Norwegian Ivar Giaever applied this method to prove in 1960 that the BCS theory of low temperature superconductivity is correct. It had assumed that electrons can constitute bound pairs and predicted the existence of a forbidden band for possible energies. Giaever shared the Nobel Prize with Esaki 
for demonstrating that this is true.

Somewhat later, physicists realized that it is not necessary to apply a potential difference when the passage of electrons through a very thin gap between two semiconductors is not possible. It is sufficient that electrons of higher energy lose some kinetic energy inside the gap by collisions with other particles. Turin understood that this method allows us to distinguish molecules from one another, since this is equivalent to determining the energy required to excite vibrations inside these molecules. This is a simplified version of infrared spectroscopy, but requires specific receptors for different odors. That is a matter of genetics, as for color vision. Our color-space is usually three-dimensional, while the odor-space is multidimensional. Honeybees have 174 types of receptors and ants have even about 400 different ones [98]. This fact led to a system of communications that determines the peculiar structure of these societies. There is no privacy. Information is locally shared.

\subsection{The Nature of Signals Discovered by Benveniste}

Dr. Benveniste discovered the existence of water memory, but this phenomenon could not yet be explained in 1988. Since his experimental results were attributed to error or fraud, he was constantly searching simpler and more objective methods to prove the reality of water memory. In 1990, he began to use the system of Langendorff ([6], p. 123). It is based on an allergic reaction of isolated hearts of guinea pigs or rats and modifies the coronary flow by about 20\% ([10], pp. $337-343,354)$. He used empirical methods, but had heard about the theory of Coherent Domains, where EM fields seemed to be involved. He tried thus to verify if magnetic fields of $50 \mathrm{~Hz}$, easily produced by means of the European electric distribution system, might have some effect. About 100 experiments were performed in 1990 and 1991 with physicists of the Central Laboratory of Magnetism in France. It appeared that these fields could annihilate the biological efficiency for EHDs of histamine ([6], pp. 126-128).

This fact suggested that EM signals might be involved in water memory, but how could that be proven? Benveniste spoke in 1992 to a friend, who was an electronics hobbyist and thought that if molecules are able to produce EM waves, it might be possible to detect them by means of a coil. He constructed a kit, used for amplifying telephone sounds. It turned out that this method was sufficient to detect signals, created by an EHD that was contained in a tube, simply placed inside a coil. The wire delivered an electric signal that could be amplified ([6], pp. 128-132) by means of an electronic circuit that is valid for microphones, detecting audible sound waves. The detected signal varied thus at low frequencies (20 $\mathrm{Hz}-20 \mathrm{kHz}$ ). When the amplified signal was applied to a second coil and when another tube, containing only pure water, was placed in this coil, it acquired the capacity to stimulate the same receptors than the original EHD. This fact did prove that EHDs produce "signals" that allow for information transfer. Beauvais called this a "molecular telephone" ([10], p. 336). 
It was not possible anymore to attribute the published results to errors or sloppy work. Benveniste tried thus to restore his credibility in 1993 by means of experiments, performed with the system of Langendorff and controlled by physicists in Georges Charpak's laboratory in Paris. Charpak told Benveniste that if molecular communications were possible by means of ELF waves, that "would be the biggest discovery since Newton, if it were true". However, he was convinced that some fakers in Benveniste's laboratory did "arrange" the experimental results ([6], pp. 155-157). Nevertheless, it was decided that two types of molecules (acetylcholine and ovalbumin) should be used for preparing EHDs. They would be blindly treated by means of coding, but the results were catastrophic. For 11 of 18 series of tests performed in 1994, the reaction of the isolated hearts was inversed. Authentic EHDs had no biological efficiency anymore, while pure water produced positive results.

Benveniste tried to understand this fact. He knew that the tubes had not been exchanged, since he did transport them himself, but they had been placed side by side. The capacity of genuine EHDs to provoke biological reactions could thus have been transferred to pure liquid water, even without needing intermediate detection and amplification. To test this hypothesis, he did shield all samples by means of thick aluminum foil, but such a Faraday cage was not sufficient to suppress the unexpected information transfer. We conclude that it was not merely due to oscillating electric fields, but for Charpak, the idea of information transfer from tube to tube was even more abstruse than water memory. Actually, he wrote in July 1995 to Benveniste that he advances "the most baroque reasons to explain the failures" ([6], p. 166). This reaction was revolting for Benveniste. He was accustomed to attach primary importance to experimental evidence, but was now confronted with the conviction that "a phenomenon that cannot be explained with present-day knowledge cannot exist." ([6], p. 90).

Benveniste considered, on the contrary, that the new, objectively established facts demonstrated that information transfer is possible. It has to result from signals, which could be "intrinsic to molecular activity" ([6], p. 164). How this might be achieved remained mysterious, but Benveniste continued to improve his experimental methods ([13], pp. 336, 461, 519, 542, 584). His now very small laboratory acquired in 1995 a computer with a program for sound analysis. The electric signals, obtained by means of a coil could be digitalized before applying them to the second coil ([10], p. 461). Pure water became active, when it was exposed to this signal, even when it was digitally transmitted between different continents. He described these results in an article, submitted for publication to the Journal of Immunology. The final answer was that "the protocol and experiments are perfect, but the article cannot be published without precisions concerning the exact physical nature of the signal" ([6], p. 179).

It was only known that molecules can emit and absorb EM waves in the frequency domain of microwaves or infrared light. That molecules are able to emit signals of very low frequencies seemed to be impossible. Moreover, it was believed that biologically active molecules can only stimulate their receptors 
according to the model of chemical reactions. Since water memory transgressed this dogma, the well-known neurologist Changeux called it a "scientific heresy" ([6], p. 194). Why did referees and "experts" not verify if the reported facts are true? Why did the physicist Charpak not help to identify the nature of the signals? Benveniste recognized that "classical biologists" are not competent in electro-physics, since they use "structural models" ([6], pp. 180, 184). He mentioned also that he had only "adopted the hypothesis of an electromagnetic nature of the molecular signal" to organize empirical research ([6], p. 211). He was scientifically isolated and Charpak told him even: "your experiments challenge elementary laws of physics and common sense" ([6], p. 212). As far as we know, the nature of the relevant signals has never been identified. We summarize our explanation in Figure 34. The upper line corresponds to the description that we used so far. Electrically charged parts of active molecules do create an oscillating electric field.

We could compare the frequency f of the oscillating electric charge of active molecule to the message that has to be transmitted to potential receptors. This can be done in a direct way or by means of a bypass, as indicated in Figure 20. Trimmed chains are then the information carriers and could be compared to messengers. Anyway, the information has to be expressed or encoded in a particular way. For usual language, it could be encoded in oral or written form. For water memory, the vehicle can be an oscillating electric field, but this is not the only possibility. This results from the fact that oscillating electric charges are equivalent to electric currents. They create an oscillating magnetic field. [Initially, Oersted did use Volta's pile to show that motions of electric charges inside a wire do heat this conductor, but he noted that the current caused a rotation of a nearby compass needle. This phenomenon was totally unexpected, since it was only known that magnetic materials are able to do that. Oersted described in 1820 the properties of the new type of "magnetic forces" in terms of actions at a distance, as Newton did for gravitational forces and Coulomb for electric forces.]

The concepts of electric and magnetic fields were introduced by Faraday. [He discovered in 1831 that imagined "lines of magnetic forces" passing through a closed loop of a conductive wire induce there an electric current, but only when the flux is varying. It can increase, decrease or oscillate. In 1845 , he used the

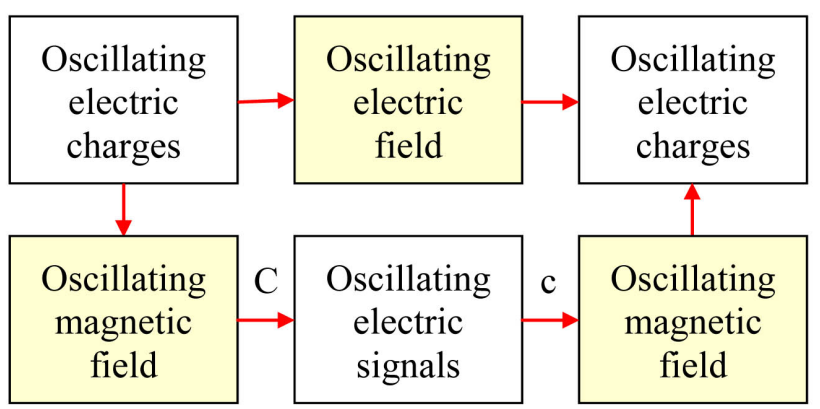

Figure 34. Communications by means of oscillating electric and magnetic fields inside EHDs, with the aid of two coils ( $\mathrm{C}$ and $\mathrm{c}$ ). 
more general concept of electric and magnetic fields, defined for any point in space and time by means of a fictitious experiment. These fields can then vary in space and time.] The second line of Figure 34 shows that oscillating electric fields can be replaced by oscillating magnetic fields. For Benveniste and Montagnier these fields were hidden in "black boxes". They become now mentally transparent and even brilliant. Charpak could have explained what was in these black boxes, but did not even care, since they were irrelevant for him.

The second line of Figure 34 indicates also that when a tube with an EHD of a particular type of active molecules is placed inside a coil C, the oscillating magnetic field exerts a force on charged particles in the wire. This force produces a current when the circuit is closed or a tension between the extremities of an open circuit. [This fact results from Faraday's discovery.] The resulting electric signal can be amplified and applied to a second coil c. It produces a current in the wire of this coil and thus again a magnetic field. It oscillates at the same frequency and exerts a force on electric charges. Benveniste compared the relevant signals to radio waves. This analogy was helpful, but they were merely electric and magnetic fields, oscillating at low frequencies. They require the presence of electric charges, while EM waves consist of electric and magnetic fields that are constantly transformed into one another and can even propagate in vacuum. They are characterized by the relation $f=c / \lambda$, where the velocity $c \approx 3 \times 10^{8} \mathrm{~m} / \mathrm{s}$.

\subsection{Stimulation by Low-Frequency Magnetic Fields}

After realizing that water memory is transferable to pure water, Benveniste developed "digital biology" with the engineer Didier Guillonnet, who joined the team in 1996 ([13], p. 596). Their common patent [99] indicates that information transfer is even possible when pure water is simply flowing through the second coil (c). This means that the formation of new trimmed chains of WPs is a rapid process. It would even be sufficient to create one trimmed chain of WPs, since it will generate other equally trimmed chains of WPs. This fact is also important for beat phenomena, since equally trimmed chains of WPs are then reproduced inside the initially pure water. Benveniste had noted that shaking of the receiving tubes was not required, but Benveniste and Guillonnet found that "it is possible to improve the performance" of this system by adding a signal that corresponds to white noise. It covered the whole low frequency domain $(1 \mathrm{~Hz}$ to $20 \mathrm{kHz}$ ). They mentioned that they were surprised when they discovered this fact. Konovalov and Ryzhkina noted also that the formation of supramolecular structures is favored by low-frequency EM fields [87].

Montagnier and his collaborators [14] placed the test tubes, which contain "informed" and "naive" water in different coils ( $\mathrm{C}$ and $\mathrm{c}$ ), but inside a greater coil (C'). It was subjected to an electric current, oscillating at $7 \mathrm{~Hz}$. This frequency was chosen since it is close to "Schumann frequencies". They correspond to genuine $E M$ waves, generated by thunderstorms in the terrestrial atmosphere and reflected by the surface of the Earth and the ionosphere. Since these waves 
are propagating in a spherical layer, they correspond to standing waves and their wavelength $\lambda$ is of the order of the circumference of the Earth. It follows that their frequency $f=\mathrm{c} / \lambda \approx 300$ (thousand $\mathrm{km} / \mathrm{s}$ ) $/ 40$ (thousand $\mathrm{km}$ ) $=7.5 \mathrm{~Hz}$. The measured fundamental frequency for Schuman resonances is close to $7.8 \mathrm{~Hz}$. However, the coil C' creates only an oscillating magnetic field and not an EM field, similar to radio waves of extra low frequencies.

The term of "electromagnetic fields" was probably used by Benveniste and Montagnier in the general sense of being related to electric and magnetic phenomena. To realize the difference, we note that standing waves on trimmed chains of WPs could be compared to standing waves for oscillating electrons in an antenna that radiates EM waves. Their wavelength $\lambda$ would then be determined by the length of these chains $(L=\lambda / 2 \approx 300 \mathrm{~nm})$, but their frequency would be extremely high $\left(f=c / \lambda \approx 5 \times 10^{14} \mathrm{~Hz}\right)$. The inverse process corresponds to an EM wave that excites standing waves for oscillating electrons in a receiving antenna. For $7 \mathrm{~Hz}$, the wavelength $\lambda=c / f \approx 43,000 \mathrm{~km}$.

Nevertheless, a coil C' and low frequency currents were beneficial. To explain this fact, we recall that water molecules are bound to one another in bulk liquid water by hydrogen bonds (Figure 4(a)), but are constantly exchanged at the time scale of $10^{-12} \mathrm{~s}$. The applied magnetic field is oscillating at a much lower frequency $(7 \mathrm{~Hz}$ or $1 \mathrm{~Hz}-20 \mathrm{kHz})$ and does reorient the effective dipoles of water molecules at this frequency. They will thus be liberated from their usual bonds. This facilitates the formation of water pearls and trimmed chains of WPs.

We recall also that "wild transfers" were not suppressed when the test tubes were shielded by means of aluminum foil. It did only suppress electric fields. That was not sufficient, since magnetic fields do also allow for information transfer. However, it is possible to eliminate magnetic fields by means of mu-metal. Benveniste had already discovered that it abolishes crosstalk and beat phenomena ([10], p. 565) and Montagnier [14] did interpose a sheet of mu-metal between the primary coil $\mathrm{C}$ and the secondary coil c. Empirical discoveries do not require that their cause is already known.

\subsection{Resonances for Pairs and Chains of Water Pearls}

We come now back to the important and still unsolved problem of the asymmetry of the energy-loss functions for Mahata's resonances (Figure 33). The model of single WPs has to be corrected, since WPs would be subjected to Brownian motion. They attract one another and will thus constitute pairs of WPs. Oscillatory rotations are then possible for both WPs, even at a frequency $\Omega \approx 25 \mathrm{MHz}$ ), because of surrounding water molecules. However, mutual attraction of neighboring poles would yield an additional restoring force, which could have noticeable effects.

To acquire more physical insight, we consider two identical pendulums (Figure 35(a)). They would oscillate at the same frequency $\Omega$, but can be coupled by means of a weak spring. [A normal string is sufficient, when it is somewhat longer than the separation of the masses at rest and when a small mass is attached to 


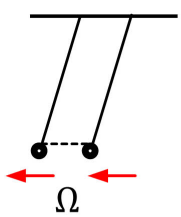

(a)

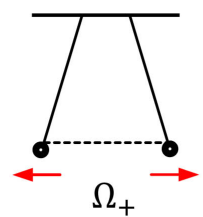

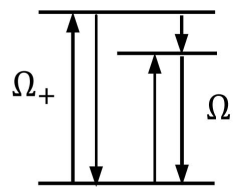

(b)

Figure 35. (a) Low and high frequency modes for free oscillations of two coupled pendulums; (b) Resonances correspond to quantum mechanical transitions, allowing for excitation at the frequency $\Omega_{+}$and emission at the frequency $\Omega$.

the middle of the string.] Free motions of the coupled pendulums depend on the chosen initial conditions and can be quite complicated, but there are two special cases, shown in Figure 35(a). It is intuitively clear that both masses will oscillate at the frequency $\Omega$ for strictly parallel motions, since the length of the intermediate spring is not modified. It is higher for antiparallel motions $\left(\Omega_{+}>\Omega\right)$. These coordinated motions are called normal modes of oscillation, since the system behaves like a single oscillator. It is possible to produce forced motions of both pendulums, by applying an oscillating force to one of them.

Figure 35(b) recalls that $\mathrm{QM}$ is a generalization of classical mechanics. The lowest possible energies for any single harmonic oscillator are then separated by intervals that are proportional to the resonance frequency. This can be $\Omega$ or $\Omega_{+}$. Classically described forced oscillations are then replaced by transitions. It should thus be possible to absorb EM energy at the frequency $\Omega_{+}$and to restitute a part of it at the lower frequency $\Omega$. This happens for fluorescence and Mahata's energy-loss functions $\beta_{2}(\omega)$ displayed always at the side of lower frequencies (Figure 33).

Figure 36 shows that we get also two normal modes for small oscillatory rotations of the coupled WPs. As for Figure 35(a), displacements of neighboring poles can be parallel or antiparallel. They are perpendicular to the symmetry axis and represented by red arrows. To account for possible resonances, we have to consider two coupled equations of motion:

$$
\begin{gathered}
\ddot{u}_{1}+\Omega^{2} u_{1}+\omega_{o}^{2}\left(u_{1}-u_{2}\right)+\gamma_{1} \dot{u}_{1}=C_{\omega} \exp (-i \omega t) \\
\ddot{u}_{2}+\Omega^{2} u_{2}-\omega_{o}^{2}\left(u_{1}-u_{2}\right)+\gamma_{2} \dot{u}_{2}=-C_{\omega} \exp (-i \omega t)
\end{gathered}
$$

They apply to the left part of Figure 36 and $\Omega \approx 25 \mathrm{MHz}$ is the resonance frequency for independent oscillations, while $\omega_{o} \approx 1 \mathrm{kHz}$ accounts for interactions. They correspond to opposite forces. For the right part of Figure 36, it is sufficient to change the sign of $u_{2}$. Both water pearls are subjected to viscous friction, but it is not identical, since the resulting motions of the surrounding liquid water can hinder or facilitate one another. To assume that $u_{1}>u_{2}$ implies that $\gamma_{1}<$ $\gamma_{2}$. When we set $\gamma_{1}=\gamma-\eta$ and $\gamma_{2}=\gamma+\eta$, the sum and the difference of (19) and (20) yield two equations for $u_{ \pm}=u_{1} \pm u_{2}$ :

$$
\begin{gathered}
\ddot{u}_{+}+\Omega^{2} u_{+}+\gamma \dot{u}_{+}-\eta \dot{u}_{-}=0 \\
\ddot{u}_{-}+\Omega_{+}^{2} u_{-}+\gamma \dot{u}_{-}-\eta \dot{u}_{+}=2 C_{\omega} \exp (-i \omega t)
\end{gathered}
$$




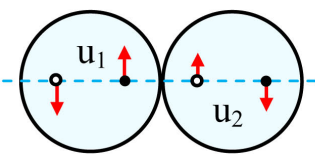

$\Omega$

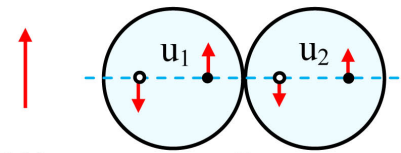

$\Omega_{+}$

Figure 36. Two modes of forced motions for pairs of WPs.

The pair of WPs behaves thus as if there were two oscillators that resonate at the frequencies $\Omega$ or $\Omega_{+}$, where $\Omega_{+}^{2}=\Omega^{2}+2 \omega_{o}^{2}$. However, only the mode $u_{-}$is directly excited by the oscillating electric field of angular frequency $\omega$. The mode $u_{+}$is then excited by entrainment, but forced oscillations require that

$$
u_{ \pm}=A_{ \pm} 2 C_{\omega} \exp (-i \omega t)
$$

Because of (21) and (22), the amplitudes $A_{ \pm}$are determined by the equations:

$$
a_{+} A_{+}+i \omega \eta A_{-}=0 \text { and } a_{-} A_{-}+i \omega \eta A_{+}=1
$$

where $a_{+}=\Omega^{2}-\omega^{2}-i \omega \gamma$ and $a_{-}=\Omega_{+}^{2}-\omega^{2}-i \omega \gamma$. Thus,

$$
A_{+}=-\frac{i \omega \eta}{a_{+}} A_{-} \text {and }\left[a_{-}+\frac{(\omega \eta)^{2}}{a_{+}}\right] A_{-}=1
$$

Forced oscillatory rotations of pairs of WPs involve both modes of oscillation. The polarizability is thus

$$
\beta(\omega)=\Omega_{+}^{2}\left(A_{-}+A_{+}\right)
$$

It is normalized to get $\beta(0)=1$. When $\Omega$ and $\omega_{o}$ correspond to $25 \mathrm{MHz}$ and 1 $\mathrm{kHz}$, there are only two adjustable parameters: $\gamma$ and $\eta$. They specify the average energy loss and energy transfer by viscous friction. The resulting values of $a_{ \pm}$and $A_{ \pm}$allow us to calculate the spectral distribution of the real and imaginary parts of $\beta(\omega)$. When $\gamma=20$ and $\eta=0.01(\mathrm{MHz})$, the calculated spectral distributions of $\beta_{1}(\omega)$ and $\beta_{2}(\omega)$ are shown in Figure 37. The energy-loss function presents a dip, like the experimentally observed one (Figure 33). The concept of water pearls accounts thus also for the asymmetry for pairs of WPs.

However, Mahata found similar curves for EHDs of various types of active molecules. This would not be compatible with the excitation of a standing wave, if all positive poles were displaced along the direction of the applied electric field E. However, the lowest possible excitation energy would only require that the rotation of one water pearl is inversed. Figure 38 shows an example. Even the excitation of standing waves on trimmed chains of WPs at high frequency and the asymmetric resonances can thus be explained by the proposed theory.

\subsection{The Stabilizing Effect of Lactose}

Homeopathic preparations are often presented in the form of pills of lactose. Opponents of this medical practice claim that lactose is merely used because of its sweet taste. Initially, Hahnemann used lactose for grinding hard substances to reduce their concentration before dissolving them in pure liquid water. Mahata 


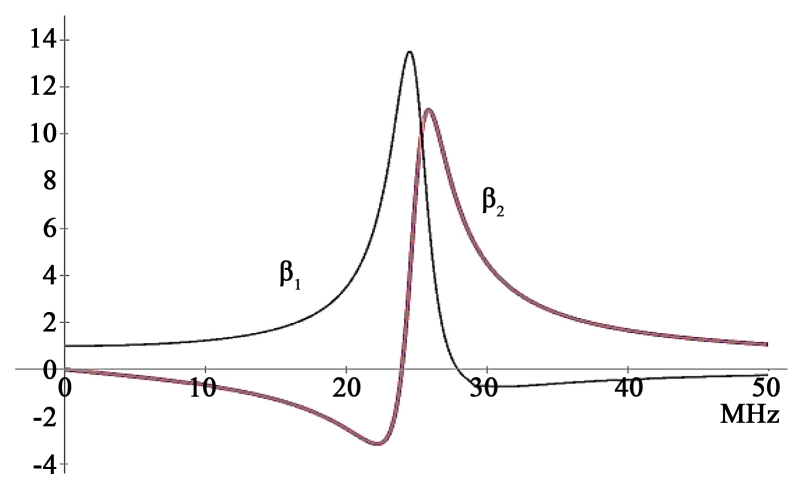

Figure 37. Predicted spectral distribution of the realpart $\left(\beta_{1}\right)$ and the imaginary part $\left(\beta_{2}\right)$ of the polarizability of pairs of coupled water pearls in pure water.

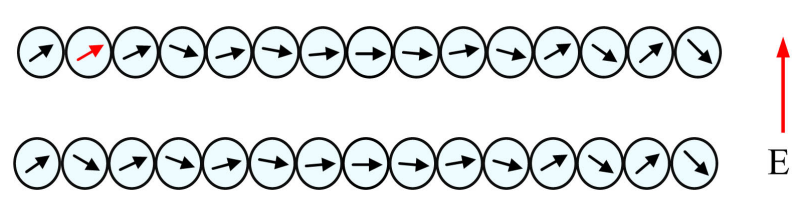

Figure 38. Resonance for trimmed chains of WPs.

knew that lactose is also used to insure better preservation of the biological efficiency of EHDs. He wanted thus to find out if the association of EHDs with lactose modifies the measurable resonance curves. When he dissolved lactose powder in pure water, he found a resonance at $50 \mathrm{MHz}$ and the energy-loss function was symmetric [100]. We have thus also to explain these facts. From the point of view of condensed matter physics, lactose is an insulator. It attracts thus charged particles by image forces. Since lactose has many pores, nearly all single WP would be attached to lactose, but the applied electric field would simultaneous set the WP and its image in oscillation. They are subjected to identical restoring forces. The resonance frequency will thus be doubled, but attachment of single WPs would keep the resonance symmetric, as for the red curve in Figure 33.

When Mahata added EHDs of Cu-Met-30 to the dissolved lactose powder, the resonance frequency was reduced to about $45 \mathrm{MHz}$, but the peak of the energy-loss function remained symmetric. Because of image forces, the trimmed chains of WPs would usually be parallel to the surface of the insulator. This will also lead to a high resonance frequency, but its value would be somewhat lower than $50 \mathrm{~Hz}$. The essential result is, of course, that the protective role of lactose is due to adhesion by image forces.

\subsection{A New Type of Molecular Interactions and Medical Applications}

The empirical discovery of water memory and its elucidation modify the traditional paradigm that molecular interactions are only possible according to the "key and slot" model of chemical reactions. Even normal interactions between biologically active molecules and their specific receptors are due to oscillating 
electric fields and resonance effects (upper lines of Figure 20 and Figure 34). Dr. Hahnemann wanted only to select medications in a rational way and to increase safety of medical practice. Dr. Benveniste did prove that this is due to the phenomenon of water memory. He asked INSERM several times that "interdisciplinary teams" could come to help him to interpret what he observed. Scientific institutions should have provided intellectual and materiel assistance, instead of "isolating" him. Benveniste felt that these authorities did even "strangle" him ([10], p. 89). The case of water memory has to be remembered as a monument of what should not happen, especially in science.

Moreover, it is not unusual that new phenomena are discovered without understanding their cause. We mentioned many examples that illustrate this fact. Christian Huyghens discovered in 1665 that pendulum clocks tend to be synchronized, but this phenomenon has only recently been explained [101]. Collective oscillations are also very important in plasma physics and provide for instance the key for understanding the occasional appearance and peculiar properties of ball lightning [102]. Oscillations of the charges of biologically active molecules and standing waves on trimmed chains of water pearls create electric and magnetic fields that tend also to be synchronized. This favors the autocatalytic generation of new trimmed chains and does more effectively stimulate the relevant receptors.

Since oscillating electric charges do also produce oscillating magnetic fields (Figure 34), we could try to pursue investigations by means of modern magnetometers. Squids are used for magneto-encephalography, but a new, less expensive and very efficient technology is now available in the form spintronics. This word is an abbreviation of spin-electronics. Instead of exerting forces on the electric charge of electrons, it is indeed possible to exert also forces on their spin. This technology has medical applications [103]. New magnetic detectors of this type have a very high sensitivity in a large frequency domain (in the picotesla region for frequencies below $100 \mathrm{~Hz}$ ). Their small active areas allow for unprecedented spatial resolution (down to tens of nanometers). They seem thus to be ideal tools for trying to study interactions between active molecules and their receptors. They could open new avenues for future research in biology, medicine and pharmacology, if it were possible to detect low resonance frequencies of biologically active molecules and their specific detectors.

This has already been achieved by means of EHDs and there are observations that indicate the usefulness of such measurements. The collaboration of Mahata with the medical doctor Chattopadhyay, an Indian specialist of homeopathy, led indeed to are markable discovery [104] [105]. They found that EHDs of the blood serum of patients did also produce resonances at about $25 \mathrm{MHz}$. They were similar to those of EHDs of biologically active molecules. This proves that they have charged parts that can also oscillate and create trimmed chains of WPs. Moreover, Chattopadhyay found that for patients, having ailments that can be treated by means of homeopathic medicines, preparations that led to a closer match of resonance curves were more efficient. It was concluded that it 
should be possible to "make a selection of medicines based on scientifically measurable parameters." The reported results require independent confirmations, but it would not be prudent to discard this possibility without further objective studies.

The basic claim of Montagnier's patent US2010323391 [47] was that hidden infections in humans and animals can be detected by a non-invasive method. It requires only to collect blood samples and to prepare EHDs of them. This allowed for spectral analysis of signals that were detected by means of coils. Another patent added that "any human or animal fluid, e.g. blood, urine, various secretions" can be used for this purpose. Even the unexplained resurgence of some sicknesses might result from water memory, since Montagnier [14] [15] found that DNA molecules do also produce similar signals.

Another potentially important question concerns Hahnemann's empirical rule that "like cures like". Is it possible to prove the existence of a link between receptors and the sickness they can cure? Present-day knowledge and already acquired experience in the domain of receptors and neurology could be used, for instance, to verify if some sicknesses are related to particular receptors, by exiting them. Research is motivated by curiosity, whatever may be found.

\section{Conclusions}

The objective of this study was to find out if water memory is possible or not. We treated this problem in terms of condensed matter physics, but it illustrates a much more general and fundamental difficulty: the recurrent conflict between facts and ideology. In science, it is not unusual that empirical research uncovers phenomena without understanding the underlying mechanism. It does even happen that the framework of existing theories cannot explain them. Past experience and commonsense tell us that previous assumptions may have to be corrected in such a situation. Kuhn has shown [45] that the discovery of an anomaly with respect to "normal theory" does often lead to initial rejection. This was also true for water memory. It is thus necessary to be aware of the fact that scientific progress can be drastically slowed down by so-called "skeptics", who are not skeptic enough in regard to their own preconceptions and beliefs.

It is true that "extraordinary claims require extraordinary evidence," but this slogan does only displace the basic problem. What is valid evidence? In science, it can merely be recognized by referring to reality. This is well-known, but unfortunately, there is a strong tendency to rely only previously acquired ideas and theories, although they could have been based on hypotheses that were only valid in a limited domain. They may have to be replaced by more general ones. The case of water memory illustrates such a need in a rather exemplary way.

Physics is also confronted today with a similar problem. It results indeed from observations that there are only certain types of elementary particles and that our Universe contains an enormous amount of Dark Matter. The accelerated expansion of space is caused by Dark Energy, but we are unable to explain all these facts. It is thus necessary to ask if present-day theories do not contain an 
assumption that was simple and useful, but merely an approximation. In this regard, we learned even from the development of the theory of relativity and quantum mechanics that Nature can impose restrictions on our measurements. It appeared that they are related to the existence of two universal constants ( $c$ and $h$ ). Nevertheless, we continue to believe that space and time are continuous. This is equivalent to postulating that there is no finite limit for the smallest measurable distance. How do we know? We can only say that until now, we did not yet meet such a limit. However, we can try to find out what would happen if there did exist a universally constant quantum of length (a) and thus also a universally constant quantum of time $(c a)$.

The value of $a$ is surely very small, but we cannot assert that $a=0$. If this value were finite, all physical laws for particles and fields that involve variations in space and time would not be expressed anymore by differential equations, but by finite-difference equations. [The differential wave Equation (7) was also an approximation of the more general finite-difference Equation (6), but for another reason.] When we did that for any type of particle and force fields, it turned out that the generalization would not lead to logical inconsistencies when $a \neq 0$. However, some ideas have to be changed. The highest possible energy, which has to be attributed to the whole Universe, would be finite. The behavior of fields at the smallest possible scale in space and time would be described by hitherto unknown quantum numbers. They account for all possible types of elementary particles, in agreement with already known facts [106]. This applies also to Dark Matter. It is known to be present in our Universe, but is composed of particles that have not yet been identified by means of accelerators. In cosmology, it is also possible to account for the accelerated expansion of space and Dark Energy [107]. This enlargement of the foundations of physics widens the domain of possibilities, but the concept of a "space-time continuum" is so deeply rooted in our minds and our culture, that it will take time to be able to modify this idea.

This is also true for the conviction that biologically active molecules can only interact with their specific receptors according to the model of chemical reactions. The possible existence of water memory seemed to be absurd, but the real problem was merely that it could not yet be explained. Of course, ferroelectric crystallites of water molecules and trimmed chains of water pearls are merely concepts. These entities are not directly observable, but they allow us to make verifiable predictions. An increasing part of science follows this pattern. Theory and experiment are complementary. On one hand, we have to imagine what might be possible, to draw logical consequences from the proposed hypotheses. On the other hand, we can establish what it real or not. Sometimes the observations precede their explanation and sometimes, they can be used to test the validity of hypothesis and theories.

In regard to water memory, we found that the concept of trimmed chains of WPs accounts for the quasi-periodic variations of the biological efficiency of EHDs (Sections 3.4 and 3.5), the measurable frequency spectrum (Section 4.1) 
and the peculiar angles for junctions of large-scale structures (Section 4.2). It is at least probable that very long chains of WPs explain the stability of liquid water bridges (Section 4.3). The von Grotthuss mechanism can be understood in terms of intramolecular exchange effects for delocalized protons (Section 2.5). They are also relevant for $2 \mathrm{D}$ polymerization of water molecules (Section 2.6). The perplexing effects of cross talk can be attributed to beat phenomena (Section 3.7). Physicochemical and other types of measurements make sense (Sections 3.8 and 4.5). It is also possible to understand the physical nature of detected signals (Sections 5.2 and 5.3). Even Mahata's unexpected high frequency resonances can be explained (Sections 4.6, 5.4 and 5.5).

Of course, there are still open questions, inviting to pursue research (Section 5.6). We wonder for instance if low-frequency resonances can be detected for single chains of WPs. Preliminary results for dielectric responses of EHDs indicated that a resonance could be excited at about $3 \mathrm{kHz}$ [108]. Miranda did perhaps not detect them [74], since that would have required greater initial concentrations of $\mathrm{LiCl}$ or various types of biologically active molecules. The now highly developed methods of X-ray and neutron scattering could also be applied to EHDs of initially very great concentrations of ions or active molecules. We mention that Mahata obtained images of elongated entities that constituted a super lattice. These structures were observed by scanning probe microscopy [100]. Can variations of the distance between oxygen ions near the surface of water bridges subjected to different high tensions be measured diffraction of X-rays? Can oscillating magnetic fields be detected at molecular levels by means of modern magnetometers? Could water memory have contributed to the emergence of life on Earth?

Water is a very familiar substance, but still a fascinating domain of research. Moreover, it concerns not only experimental and theoretical results, but also truth and justice.

\section{Conflicts of Interest}

The author declares no conflicts of interest.

\section{References}

[1] Davanas, E., Beauvais, F., Amara, J., Oberbaum, M., Robinzon, B., Miadonnai, A., Benveniste, J., et al. (1988) Nature, 333, 816-818. https://doi.org/10.1038/333816a0

[2] Maddox, J., Randi, J. and Stewart, W.W. (1988) Nature, 334, 287-291. http://www.badscience.net/wp-content/uploads/benveniste02.pdf

[3] Maddox, J. (1988) Nature, 333, 787. https://www.nature.com/articles/333787a0 https://doi.org/10.1038/333787a0

[4] Benveniste, J., Henson, P.M. and Cochrane, C.G. (1972) Journal of Experimental Medicine, 136, 1356-1377.

https://www.ncbi.nlm.nih.gov/pmc/articles/PMC2139324/ https://doi.org/10.1084/jem.136.6.1356

[5] Benveniste, J., Egido, J. and Gutierrez-Millet, V (1976) Clinical \& Experimental 
Immunology, 26, 449-456.

https://www.ncbi.nlm.nih.gov/pmc/articles/PMC1540955/

[6] Benveniste, J. (2005) Ma vérité sur la « mémoire de l'eau. Book, Albin Michel, 223 p.

[7] Baldwin, M. (2015) The History of Medicine in Dialogue with the Present, Remedia. https://remedianetwork.net/2015/04/24/high-dilution-homeopathy-and-the-purpos e-of-the-scientific-journal/

[8] Kaufmann, A. (1994) Réseaux, 2, 183-204.

http://www.persee.fr/doc/reso_0969-9864_1994_num_2_2_3277

[9] Schiff, M. (1994) Un cas de censure dans la science: L’Affaire de la mémoire de l'eau, AlbinMichel. (1995); The Memory of Water, Thorsons, Paperback, 166 p.

[10] Beauvais, F. (2007) L'âme des molécules, une histoire de la "mémoire de l'eau", Book, Mille-Mondes; (2016) Ghosts of Molecules-The Case of the "Memory of Water". Ed. Lulu.com.

[11] Thomas, Y. (2007) Homeopathy, 96, 151-157. https://www.ncbi.nlm.nih.gov/pubmed/17678810 https://doi.org/10.1016/j.homp.2007.03.006

[12] CristianManil (2014) On a retrouvé la Mémoire de l'eau, Luc Montagnier, TV, 51.42 min. https://www.youtube.com/watch?v=_2xInJFD23k

[13] Montagnier, L. (2016) Water Memory. https://www.youtube.com/watch?v=R8VyUsVOic0

[14] Montagnier, L., Aïssa, J., Ferris, S., Montagnier, J.-L. and Lavalléee, C. (2009) Interdisciplinary Sciences, 1, 81-90. https://doi.org/10.1007/s12539-009-0036-7

[15] Montagnier, L., et al. (2010) DNA Waves and Water. https://arxiv.org/pdf/1012.5166.pdf

[16] Montagnier, L., et al. (2014) Digital Transmission of Bacterial DNA in Living Cells, UNESCO, Biology in Light of Physical Theories: New Frontiers in Medicine. https://www.youtube.com/watch?v=mYcNXjdPXGU

[17] Chaplin, M.F. (2007) Homeopathy, 96, 143-150. http://www.doktor-quak.de/wp-content/uploads/2014/01/The-Memory-of-Water-a n-overview.pdf

[18] Chaplin, M. (2016) Water Structure and Science. http://www1.lsbu.ac.uk/water/water_molecule.html

[19] Chaplin, M. (2016) Water Models. http://www1.lsbu.ac.uk/water/water_models.html

[20] https://en.wikipedia.org/wiki/Kinetic_diameter

[21] Rouard, P. and Meessen, A. (1977) Progress in Optics, VX, 77-137. http://www.meessen.net/AMeessen/optical_properties_of_thin_metal_films.pdf https://doi.org/10.1016/S0079-6638(08)70477-3

[22] Granqvistt, C.G. and Buhrman, R.A. (1976) Journal of Applied Physics, 47, 2200-2219.

https://buhrman.research.engineering.cornell.edu/pubs/JApplPhys_47_2200.pdf https://doi.org/10.1063/1.322870

[23] Latimer, W.M. and Rodebush, W.H. (1920) Journal of the American Chemical Society, 42, 1419-1433.

http://www.chemteam.info/Chem-History/Hydrogen-Bond-1920/Hydrogen-Bond1920.html

https://doi.org/10.1021/ja01452a015 
[24] Stryer, L. (1981) Biochemstry. Stanford University Freeman and Co., Text Book, 2nd Edition, 125.

[25] Chaplin, M. (2016) Ice Phases. http://www1.lsbu.ac.uk/water/ice_phases.html

[26] Mallamace, F. (2009) PNAS, 116, 1597-1598. http://www.pnas.org/content/106/36/15097 https://doi.org/10.1073/pnas.0908198106

[27] Mukhopadhyway, A., et al. (2015) Chemical Physics Letters, 623, 13-26. http://www.cchem.berkeley.edu/rjsgrp/publications/papers/2015/401_Mukhopadhy ay.pdf https://doi.org/10.1016/j.cplett.2015.04.016

[28] Köffinger, J., et al. (2011) Physical Chemistry Chemical Physics, 13, 15403-15417. https://www.ncbi.nlm.nih.gov/pmc/articles/PMC3470881/

[29] Stillinger, F.H., et al. (1978) The Journal of Chemical Physics, 69, 1473-1484. https://pdfs.semanticscholar.org/6c11/c7b39c3e2b02eb65c27e0a5600875c9128b6.pdf https://doi.org/10.1063/1.436773

[30] Von Grotthus, T. (1806) Annales de Chimie (Paris), 58, 54-73.

[31] Lippincot, E.R. and Stromberg, R. (1969) Science, 164, 1482-1487.

[32] Stromberg, J. (2013) The Curious Case of Polywater. http://www.slate.com/articles/health_and_science/science/2013/11/polywater_histo ry_and_science_mistakes_the_u_s_and_ussr_raced_to_create.html

[33] Roy, R., et al. (2009) Materials Research Innovations, 9, 98-103. https://doi.org/10.1080/14328917.2005.11784911

[34] Pollack, G. (2009) Water, Energy, and Life: Fresh Views from the Water's Edge. https://www.youtube.com/watch?v=XVBEwn6iWOo

[35] Kittel, C. (1946) Physical Review, 70, 965-971. https://pdfs.semanticscholar.org/8639/78264c8d8b616d37b96a9147b65595b61fdd.p df https://doi.org/10.1103/PhysRev.70.965

[36] Blakemore, R.P. (1975) Science, 190, 377-379. https://doi.org/10.1126/science.170679

[37] Faivre, D. and Schüller, D. (2008) Chemical Reviews, 108, 4875-4898. http://www.cup.lmu.de/ac/kluefers/homepage/L/biominerals/magnetite_2008.pdf https://doi.org/10.1021/cr078258w

[38] Eliaseev, E.A., et al. (2016) Journal of Applied Physics, 119, 204104. https://arxiv.org/abs/1602.06285 https://doi.org/10.1063/1.4952707

[39] Smith, A.M., et al. (2016) The Electrostatic Screening Length in Concentrated Electrolytes Increases with Concentration. https://arxiv.org/ftp/arxiv/papers/1607/1607.03926.pdf

[40] Lo, S.Y. (1998) Physical, Chemical and Biological Properties of Stable Water Clusters. Proceedings of the First International Symposium. http://dandylaboratories.com/wp-content/uploads/2011/09/SURVEY-OF-I-CLUST ERS.pdf

[41] HNMRC (2015) Information Paper. Evidence of the Effectiveness of Homeopathy for Treating Health Conditions, Australian Government. https://www.homeowatch.org/research/nhmrc_2015.pdf

[42] Homeopathy Research Institute (2012). http://www.nhmrchomeopathy.com/the-first-homeopathy-review.html 
[43] Shurkin, J. (2014) News Feature: Animals that Self-Medicate. http://www.pnas.org/content/111/49/1733 https://doi.org/10.1073/pnas.1419966111

[44] Hahnemann's “Organon of Medicine” $(1833,1842)$ Transl. by R.E. Dudgeon and W. Boericke. http://www.homeopathyhome.com/reference/organon/organon.html

[45] Kuhn, T.S. (1962) The Structure of Scientific Revolutions. University of Chicago Press. (1st Edition, 172 p; 2012, 4th Edition, 264 p).

[46] Elia, V and Nicoli, M. (1999) Annals of the New York Academy of Sciences, 879, 241-248. https://doi.org/10.1111/j.1749-6632.1999.tb10426.x

[47] Elia, V., et al. (2004) Journal of Thermal Analysis and Calorimetry, 78, 331-342. https://doi.org/10.1023/B:JTAN.0000042179.07858.c8

[48] Elia, V., et al. (2007) Homeopathy, 96, 163-169. http://omeopatia.org/download/seminario-rey/ELIA-Dell-acqua-la-rimembranza-i nglese.pdf https://doi.org/10.1016/j.homp.2007.05.007

[49] Elia, V., et al. (2008) Journal of Thermal Analysis and Calorimetry, 93, 1003-1011. https://doi.org/10.1007/s10973-007-8843-8

[50] Elia, V., et al. (2014) Conference on the Physics, Chemistry and Biology of Water. https://www.youtube.com/watch?v=EPVHl3xR7p0

[51] Montagnier, L. (2011) DNA “Teleportation”. http://www.rexresearch.com/montagnier/montagnier.htm

[52] Alm, J.F. and Walker, J.S. (2002) SIAM Review, 44, 457-476. https://epubs.siam.org/doi/pdf/10.1137/S00361445003822 https://doi.org/10.1137/S00361445003822

[53] Lo, S.Y. (1996) Modern Physics Letters B, 10, 921-930. https://www.cs.cmu.edu/ dst/ATG/lo-iestru.html https://doi.org/10.1142/S0217984996001048

[54] Lo, S.Y., et al. (2009) Physics Letters A, 373, 3872-3876. https://www.researchgate.net/publication/222823593_Evidence https://doi.org/10.1016/j.physleta.2009.08.061

[55] Gann, D. and Lo, S.Y. (2009) Double Helix Water. D and W Publ., 316 p.

[56] Ho, W.M. (2014) Water, 6, 1-12. http://www.waterjournal.org/volume-6/ho

[57] Armstrong, W.G. (1893) The Electrical Engineer, 10, 154-152. http://ttm-old.tugraz.at/jw/down/Armstrong_1893.pdf

[58] Fuchs, E.C., et al. (2007) Journal of Physics D: Applied Physics, 40, 6112-6114. https://doi.org/10.1088/0022-3727/40/19/052

[59] Fuchs, E.C. (2016) Protons and the Floating Water Bridge (Interview at the Conference on the Physics Chemistry and Biology of Water). https://www.youtube.com/watch?v=v8ES0Y0564M

[60] Fuchs, E.C. (2014) Protons and the Electrical Conduction in a Floating Water Bridge. Conference on the Physics, Chemistry and Biology of Water. https://www.youtube.com/watch?v=ZB1bCt9JmeE

[61] Fuchs, E.C., et al. (2016) Journal of Physics D, 49, 1-7. http://iopscience.iop.org/article/10.1088/0022-3727/49/12/125502/pdf https://doi.org/10.1088/0022-3727/49/12/125502

[62] Ponterio, R.C., et al. (2011) Journal of Physics D, 43, 175405-175412. http://iopscience.iop.org/article/10.1088/0022-3727/43/17/175405 
https://doi.org/10.1088/0022-3727/43/17/175405

[63] Skinner, L.B., et al. (2012) PNAS, 109, 16463-16468. http://www.pnas.org/content/109/41/16463.full.pdf https://doi.org/10.1073/pnas.1210732109

[64] Fuchs, E.C., et al. (2009) Journal of Physics D, 42, 105502 (10 p). https://hal.archives-ouvertes.fr/hal-00569550/document

[65] Namin, R.M., et al. (2013) Physical Review E, 88, 033019 (6 p). https://arxiv.org/abs/1210.2913

[66] Fuchs, E. (2009) The Inner Structure of a Floating Water Bridge, SETI Talks. https://www.youtube.com/watch?v=N1At3Gcd-No

[67] Del Guidice, E., Preparata, G. and Vitiello, G. (1988) Physical Review Letters, 61, 1085-1088.

https://journals.aps.org/prl/abstract/10.1103/PhysRevLett.61.1085 https://doi.org/10.1103/PhysRevLett.61.1085

[68] Preparata, G. (1995) QED Coherence in Matter. World Scientific, 252 p. https://doi.org/10.1142/2738

[69] Del Guidice, E. (2014) Water, 6, 61-71.

http://www.waterjournal.org/uploads/vol6/delgiudice/WATER.2014.3.DelGiudice.p df

[70] Del Guidice, E., et al. (2011) Journal of Physics. Conference Series, 329, 012001. http://iopscience.iop.org/article/10.1088/1742-6596/329/1/012001/pdf https://doi.org/10.1088/1742-6596/329/1/012001

[71] Bellavite, P., et al. (2013) Homeopathy, 103, 4-21. https://www.researchgate.net/publication/259805970 https://doi.org/10.1016/j.homp.2013.08.003

[72] Montagnier, L., et al. (2017) Water, 9, 339-357. https://arxiv.org/pdf/1804.02436.pdf https://doi.org/10.3390/w9050339

[73] Yinon, T.A. and Liu, Z.Q. (2015) Water, 7, 33-95.

[74] Miranda, A.R., et al. (2011) Materials Research Innovations, 15, 302-308. http://www.fap.if.usp.br/ vannucci/vannucci_H2Oarticle.pdf https://doi.org/10.1179/143307511X13109310554445

[75] Maity, T., Ghosh, D. and Mahata, C.R. (2010) Homeopathy, 99, 99-103. https://www.ncbi.nlm.nih.gov/pubmed/20471612 https://doi.org/10.1016/j.homp.2009.10.004

[76] Mahata, C.R. (2013) Homeopathy, 102, 262-3267. http://daneshyari.com/article/preview/2630007.pdf https://www.sciencedirect.com/science/article/pii/S1475491613000635?via\%3Dihub https://doi.org/10.1016/j.homp.2013.07.003

[77] Mahata, C.R. (1997) The Homeopathic Heritage, 22, 245-252.

[78] Barnard, G.P. (1965) Journal of the American Institute of Homeopathy, 58, 205-212.

[79] Barnard, G.P. and Stephenson, J.H. (1967) Journal of the American Institute of Homeopathy, 60, 277-286.

[80] Gay, A. and Boiron, J. (1953) Démonstration physique de l'existence réelle du remède homéopathique, Ed. Lab. P.H.R., Lyon, France (Communication Internal Congress in Brussels). 
[81] Maity, T., Ghosh, D. and Mahata, C.R. (2007) J. Inst. Electr. Enineers (India), 88, 27-31.

[82] Rey, L. (2003) Phyica A, 323, 67-74. http://www.homresearch.org/docs/Physica\%20A.PDF

[83] Rey, L. (2007) Homeopathy, 96, 170-174. https://doi.org/10.1016/j.homp.2007.05.004

[84] van Wijk, R., et al. (2006) Journal of Alternative and Complementary Medicine, 12, 437-443.

http://www.doktor-quak.de/wp-content/uploads/2014/01/Thermoluminescence-inUltra-High-Dilution-Research.pdf https://doi.org/10.1089/acm.2006.12.437

[85] Demangeat, J.-L. (2013) Homeopathy, 102, 87-105. https://doi.org/10.1016/j.homp.2013.01.001

[86] Van Wassenhoven, M., et al. (2017) Homeopathy, 106, 223-239. https://doi.org/10.1016/j.homp.2017.08.001

[87] Konovalov, A.L. and Ryzhkina, L.S. (2014) Geochemistry International, 52, 1207-1226. https://link.springer.com/article/10.1134/S0016702914130072

[88] Bunkin, N.F. and Shkirin, A.V. (2012) The Journal of Chemical Physics, 137, 054707. https://doi.org/10.1063/1.4739530

[89] Häbich, A., et al. (2010) The Journal of Physical Chemistry B, 114, 6962-6967. https://doi.org/10.1021/jp911868j

[90] Demangeat, J.-L. (2015) La Revue d Homéopathie, 6, 125-139. https://doi.org/10.1016/j.revhom.2015.10.002

[91] Sedlak, M. and Rak, D. (2013) The Journal of Physical Chemistry B, 117, 2495-2504. https://doi.org/10.1021/jp4002093

[92] Elia, V., et al. (2013) Water, 5, 16-26. http://www.waterjournal.org/volume-5/de-ninno

[93] Elia, V., Yinon, T.A., et al. (2016) Water, 8, 1-23.

[94] Ogle, W. (1870) Medico-Chirurgical Transactions, 53, 263-290. https://www.ncbi.nlm.nih.gov/pmc/articles/PMC2150467/ https://doi.org/10.1177/095952877005300113

[95] Dyson, G.M. (1938) Journal of Chemical Technology \& Biotechnology, 57, 647-651. https://doi.org/10.1002/jctb.5000572802

[96] Turin, L. (1996) Chemical Senses, 21, 773-791. https://academic.oup.com/chemse/article-pdf/21/6/773/1320021/21-6-773.pdf https://doi.org/10.1093/chemse/21.6.773

[97] Turin, L. (2007) The Secret of Scent: Adventures in Perfume and Science of Smell. Harper Collins, 224 p.

[98] Welcome to the Zwiebel Lab. (2012) Vanderbilt University, Ant Chemosensory Biology. http://www.vanderbilt.edu/zwiebellab/Home.html

[99] Benveniste, J. and Guillonnet, D. (2001) Method, System and Device for Producing Signals from a Substance Biological and/or Chemical Activity.

https://patents.google.com/patent/US6541978

[100] Mahata, C.R. (2018) Journal of Homoeopathy University, 3, 1-8.

[101] Oliveira, H.M. and Melo, L.V. (2015) Huygens Synchronization of Two Clocks. Scientific Reports 5, Article 11548. https://www.nature.com/articles/srep11548 https://doi.org/10.1038/srep11548 
[102] Meessen, A. (2010) Ball Lightning: Bubbles of Elecronic Plasma Oscillations. International Symposium on Ball Lightning, Kaliningrad, 21-27 June 2010. http://www.meessen.net/AMeessen/BL-Theory.pdf

[103] https://www.mddionline.com/new-spin-magnetic-sensors-medical-devices

[104] Chattopadhyay, R. and Mahata, C.R. (2016) International Journal of Complementary \& Alternative Medicine, 3, 00078 (4 p).

[105] Chattopadhyay, R. and Mahata, C.R. (2016) International Journal of High Dilution Research, 15, 11-17.

http://highdilution.org/index.php/ijhdr/article/view/818

[106] Meessen, A. (2017) Journal of Modern Physics, 8, 35-56. http://file.scirp.org/pdf/JMP_2017011015364095.pdf https://doi.org/10.4236/jmp.2017.81004

[107] Meessen, A. (2017) Journal of Modern Physics, 8, 251-267. http://file.scirp.org/pdf/JMP_2017022811200542.pdf https://doi.org/10.4236/jmp.2017.82017

[108] Li, W., et al. (1998) Dielectric Spectral Study in Low Frequency of IE Water and Some Other Solvents. Ref. [50], 175-189. 


\section{Appendix: The Size of Water Pearls}

The basic ideas were presented in Section 2.8. Since $\phi(r \pm \Delta r)=\phi(r) \pm \Delta r \phi^{\prime}(r)$, where the prime indicates derivation with respect to $\mathrm{r}$, the potential in the external medium $(r \geq R)$ is

$$
\phi_{e}(r, \theta)=-P\left(\frac{1}{r^{2}}+\frac{1}{r \lambda_{o}}\right) \cos \theta \mathrm{e}^{-r / \lambda_{o}} \quad \text { while } \phi_{i}(r, \theta)=E_{i} r \cos \theta
$$

is the potential in the internal medium $(r \leq R) . P$ is the total dipole moment of the WP and $E_{i}$ the electric field inside this nanoparticle. In the external medium it is

$$
E_{e}(r, \theta)=-\phi_{e}^{\prime}(r, \theta)=P\left(\frac{2}{r^{3}}+\frac{2}{r^{2} \lambda_{o}}+\frac{1}{r \lambda_{o}^{2}}\right) \cos \theta \mathrm{e}^{-r / \lambda_{o}}
$$

The radius $R$ is then determined by the boundary conditions:

$$
\varepsilon_{e} E_{e}(R, \theta)+\varepsilon_{i} E_{i}(R, \theta)=\sigma(\theta) \text { and } \phi_{e}(R, \theta)=\phi_{i}(R, \theta)+w(\theta)
$$

The first relation results from Gauss's law, since the electric fields point away from the interface, which carries a surface charge density $\sigma(\theta)=\sigma_{o} \cos \theta$. The second relation accounts for the dipole density $w(\theta)$. It results from the inner and external surface charge densities $\sigma_{i} \cos \theta$ and $-\sigma_{e} \cos \theta$. They are separated by a distance $\Delta R \ll R$, but $\sigma_{e}=\varkappa \sigma_{i}$ and $\sigma_{o}=\sigma_{i}-\sigma_{e}$, while $w(\theta)=\sigma_{e} \Delta R \cos \theta$ is negligible. Moreover, $\varepsilon_{i}=0$. The boundary conditions yield then the following relations:

$$
E_{i}=-P\left(\frac{1}{R^{3}}+\frac{1}{R^{2} \lambda_{o}}\right) \mathrm{e}^{-R / \lambda_{o}} \text { and }\left(\frac{2}{R^{3}}+\frac{2}{R^{2} \lambda_{o}}+\frac{1}{R \lambda_{o}^{2}}\right) \mathrm{e}^{-R / \lambda_{o}}=\frac{\sigma_{o}}{\varepsilon_{e} P}
$$

We know that $P=\underline{N} q 2 a$, where $\underline{N}=\left(4 \pi R^{3} / 3\right) / d^{\beta}$ and $\sigma_{i}=2 q / d^{2}$. Thus, $\sigma_{o} / \mathcal{E}_{e} P=$ $(1-\varkappa)\left(3 d / 4 \pi \varepsilon_{e} a R^{3}\right)$ and

$$
\left(2+2 \eta+\eta^{2}\right) \mathrm{e}^{-\eta}=0.015(1-\varkappa) \text { where } \eta=R / \lambda_{o}
$$

We ignore the value of $\mathcal{\varkappa}=\sigma_{e} / \sigma_{\vec{p}}$ but it results from the last equation that $\eta$ is nearly constant. Indeed, $\eta=9.5$ or 10.5 when $\varkappa=0.5$ or 0.8 . Even when $\varkappa=0.2$, we get $\eta=9.1$ and $\eta=11.5$, when $\varkappa=0.9$. We conclude that $R \approx 10 \lambda_{o}$. 